Supporting information for:

\title{
Coordination of Uranyl to the Redox-Active Calix[4]pyrrole Ligand
}

Greggory T. Kent ${ }^{\dagger}$, Jesse Murillo ${ }^{\ddagger}$, Guang $\mathrm{Wu}^{\dagger}$, Skye Fortier ${ }^{\ddagger}$, Trevor W. Hayton ${ }^{\dagger *}$

'Department of Chemistry and Biochemistry, University of California Santa Barbara,

Santa Barbara, CA 93106, United States

Department of Chemistry, University of Texas at El Paso, El Paso, TX 79968, United States

*To whom correspondence should be addressed. Email: hayton@chem.ucsb.edu 
Table of Contents

$\begin{array}{lr}\text { Experimental Details } & \text { S3 } \\ \text { NMR Spectra } & \text { S10 } \\ \text { X-ray Crystallographic Data } & \text { S26 } \\ \text { IR Spectra } & \text { S27 } \\ \text { References } & \text { S30 }\end{array}$




\section{Experimental}

General. All reactions and subsequent manipulations were performed under anaerobic and anhydrous conditions under an atmosphere of dinitrogen. Diethyl ether (Et $2 \mathrm{O})$, toluene, and hexanes were dried using a Vacuum Atmospheres DRI-SOLV Solvent Purification system and stored over $3 \AA$ sieves for $24 \mathrm{~h}$ prior to use. Tetrahydrofuran (THF) was distilled over calcium hydride followed by distillation over sodium benzophenone, collected, and stored over $3 \AA$ sieves for $24 \mathrm{~h}$ prior to use. THF- $d_{8}$ and $\mathrm{C}_{6} \mathrm{D}_{6}$ were stored over $3 \AA$ sieves for $24 \mathrm{~h}$ prior to use. $\left[\mathrm{UO}_{2} \mathrm{Cl}_{2} \text { (THF) }\right)_{2},{ }^{1}\left[\mathrm{UO}_{2} \mathrm{Cl}_{2}\left(\mathrm{Ph}_{3} \mathrm{PO}\right)_{2}\right],{ }^{2}\left[\mathrm{UO}_{2}(\mathrm{OTf})_{2}(\mathrm{THF})_{3}\right],{ }^{3} \mathrm{H}_{4} \mathrm{~L}(\mathrm{~L}=\mathrm{Me} 8$-calix[4]pyrrole $),{ }^{4}$ and $[\mathrm{Li}(\mathrm{THF})]_{4}[\mathrm{~L}]^{5}$ were synthesized according to previously reported literature procedures.

All NMR spectra were recorded on a Varian UNITY INOVA 500 spectrometer or an Agilent Technologies 400-MR DD2 $400 \mathrm{MHz}$ spectrometer. ${ }^{1} \mathrm{H}$ and ${ }^{13} \mathrm{C}\left\{{ }^{1} \mathrm{H}\right\}$ NMR spectra were referenced to external $\mathrm{SiMe}_{4}$ using the residual protio solvent peaks as internal standards. The ${ }^{7} \mathrm{Li}$ and ${ }^{19} \mathrm{~F}\left\{{ }^{1} \mathrm{H}\right\}$ NMR spectra were referenced indirectly with the ${ }^{1} \mathrm{H}$ resonance of $\mathrm{SiMe}_{4}$ at $0 \mathrm{ppm}$, according to IUPAC standard. ${ }^{6,7}$ IR spectra were recorded on a Nicolet 6700 FT-IR spectrometer with a NXR FT Raman Module. Elemental analyses were performed by the Microanalytical Laboratory at University of California (Berkeley, CA).

Caution! Depleted uranium (isotope ${ }^{238} \mathrm{U}$ ) is a weak alpha emitter with a half-life of $4.47 \times 10^{9}$ years. Manipulations and reactions should be carried out in a fume hood or inert atmosphere glovebox in a laboratory equipped with $\alpha$-and $\beta$-counting equipment.

X-ray Crystallography. Data for $\mathbf{1}$ and $\mathbf{3}$ were collected on a Bruker KAPPA APEX II diffractometer equipped with an APEX II CCD detector using a TRIUMPH monochromator with a Mo K $\alpha$ X-ray source $(\alpha=0.71073 \AA$ ). Data for 2 was collected on Bruker KAPPA APEX III diffractometer equipped with an APEX III CCD detector using a TRIUMPH monochromator with a Mo K $\alpha$ X-ray source $(\alpha=0.71073 \AA$ ). The crystals were mounted on a cryoloop under Paratone$\mathrm{N}$ oil, and data was collected at 100(2) K for 1, 2, and 3 using an S3 Oxford nitrogen gas cryostream. Frame exposures of $10 \mathrm{~s}$ were used for 1, 2, and 3. Data collection and cell parameter determinations were conducted using the SMART program. ${ }^{8}$ Integration of the data frames and final cell parameter refinements were performed using SAINT software. ${ }^{9}$ Absorption corrections of the data were carried out using the multi-scan method SADABS for $\mathbf{1}$ and $\mathbf{3}$ and TWINABS for 2. ${ }^{10,11}$ Subsequent calculations were carried out using SHELXTL. Structure determination was done using direct or Patterson methods and difference Fourier techniques. All hydrogen atom 
positions were idealized and rode on the atom of attachment. Structure solution, refinement, graphics, and creation of publication materials were performed using SHELXTL. ${ }^{12}$

For complex 1, one of the solvent ligand sites of Lil is occupied by a mixture of THF and $\mathrm{Et}_{2} \mathrm{O}$ in a 58:42 ratio. As a result of this disorder, the positions of these coordinated $\mathrm{Et}_{2} \mathrm{O}$ and THF molecules were constrained with the SADI command and refined isotropically. In addition, a diethyl ether solvate in $\mathbf{1}$ is disordered over two positions, in a 50:50 ratio. As a result, C100, C101, C103, C104, and O3 were constrained using the SADI command and refined isotropically. Hydrogen atoms were not assigned to these disordered carbon atoms.

The toluene solvate in complex 2 exhibited unresolved positional disorder. As a result, its carbon atoms were refined isotropically.

Synthesis of $[\mathbf{L i}(\mathbf{T H F})]_{2}\left[\mathbf{L}^{\Delta}\right]$ (1). To a colorless stirring solution of $[\mathrm{Li}(\mathrm{THF})]_{4}[\mathrm{~L}]$ (530 mg, $0.716 \mathrm{mmol})$ in THF $(10 \mathrm{~mL})$ was slowly added $\mathrm{I}_{2}(181.6 \mathrm{mg}, 0.716 \mathrm{mmol})$. This resulted in a rapid color change to deep orange. No solid was observed in the reaction mixture. After 5 min, the volatiles were removed in vacuo to provide a deep orange solid. The solid was suspended in cold $\left(-25^{\circ} \mathrm{C}\right)$ THF $(3 \mathrm{~mL})$, and the resulting suspension was filtered through a medium porosity glass frit to provide an orange powder and a pale orange supernatant. The orange powder was subsequently rinsed with $2 \mathrm{~mL}$ of cold THF to afford $[\mathrm{Li}(\mathrm{THF})]_{2}\left[\mathrm{~L}^{\Delta}\right](\mathbf{1})$ as an orange powder. Yield: $304 \mathrm{mg}, 73 \%$. Anal. Calcd for $\mathrm{C}_{36} \mathrm{H}_{48} \mathrm{~N}_{4} \mathrm{Li}_{2} \mathrm{O}_{2}: \mathrm{C}, 74.21 ; \mathrm{H}, 8.30 ; \mathrm{N}, 9.62$. Found: $\mathrm{C}$, 73.84; H, 8.04; N, 9.32. ${ }^{1} \mathrm{H}$ NMR (500 MHz, $25^{\circ} \mathrm{C}$, THF-d8): 7.60 (br s, $\left.\mathrm{H}_{\mathrm{pyr}}, 2 \mathrm{H}\right), 6.83$ (br s, $\mathrm{H}_{\mathrm{pyr}}, 2 \mathrm{H}$ ), 5.67 (br s, $\left.\mathrm{H}_{\mathrm{pyr}}, 4 \mathrm{H}\right), 1.53$ (br s, $\mathrm{CH}_{3}, 15 \mathrm{H}$ ), 1.42 (br s, $\left.\mathrm{CH}_{3}, 6 \mathrm{H}\right), 1.29$ (br s, $\mathrm{CH}_{3}, 3 \mathrm{H}$ ). (500 MHz, $0^{\circ} \mathrm{C}$, THF- $d_{8}$ ): 7.62 (br s, $\mathrm{H}_{\mathrm{pyr}}, 2 \mathrm{H}$ ), 6.84 (br s, $\mathrm{H}_{\mathrm{pyr}}, 2 \mathrm{H}$ ), 5.67 (br s, $\mathrm{H}_{\mathrm{pyr}}, 4 \mathrm{H}$ ), 1.63 (s, $\left.\mathrm{CH}_{3}, 3 \mathrm{H}\right), 1.59$ (s, $\left.\mathrm{CH}_{3}, 6 \mathrm{H}\right), 1.51$ (s, $\left.\mathrm{CH}_{3}, 6 \mathrm{H}\right), 1.42$ (s, $\left.\mathrm{CH}_{3}, 6 \mathrm{H}\right), 1.29\left(\mathrm{~s}, \mathrm{CH}_{3}, 3 \mathrm{H}\right) .{ }^{1} \mathrm{H} \mathrm{NMR}$ $\left(500 \mathrm{MHz},-2{ }^{\circ} \mathrm{C}\right.$, THF- $\left.d 8\right): \delta 7.63\left(\mathrm{~d}, J=4.7 \mathrm{~Hz}, \mathrm{H}_{\mathrm{pyr}}, 1 \mathrm{H}\right), 6.84\left(\mathrm{~d}, J=4.9 \mathrm{~Hz}, \mathrm{H}_{\mathrm{pyr}}, 1 \mathrm{H}\right), 5.67$ (s, $\left.\mathrm{H}_{\mathrm{pyr}}, 2 \mathrm{H}\right), 5.63$ (s, $\left.\mathrm{H}_{\mathrm{pyr}}, 2 \mathrm{H}\right), 1.63$ (s, $\left.\mathrm{CH}_{3}, 3 \mathrm{H}\right), 1.61$ (s, $\left.\mathrm{CH}_{3}, 3 \mathrm{H}\right), 1.58\left(\mathrm{~s}, \mathrm{CH}_{3}, 3 \mathrm{H}\right), 1.51$ (s, $\left.\mathrm{CH}_{3}, 6 \mathrm{H}\right), 1.42$ (s, $\left.\mathrm{CH}_{3}, 6 \mathrm{H}\right), 1.29$ (s, $\left.\mathrm{CH}_{3}, 6 \mathrm{H}\right) .{ }^{1} \mathrm{H}$ NMR $\left(500 \mathrm{MHz},-40{ }^{\circ} \mathrm{C}\right.$, THF- $\left.d_{8}\right): \delta 7.64(\mathrm{~d}$, $\left.J=4.5 \mathrm{~Hz}, \mathrm{H}_{\mathrm{pyr}}, 2 \mathrm{H}\right), 6.84\left(\mathrm{~d}, J=4.8 \mathrm{~Hz}, \mathrm{H}_{\mathrm{pyr}}, 2 \mathrm{H}\right), 5.66\left(\mathrm{~d}, J=2.4 \mathrm{~Hz}, \mathrm{H}_{\mathrm{pyr}}, 2 \mathrm{H}\right), 5.62(\mathrm{~d}, J=$ $\left.2.7 \mathrm{~Hz}, \mathrm{H}_{\mathrm{pyr}}, 2 \mathrm{H}\right), 1.64\left(\mathrm{~s}, \mathrm{CH}_{3}, 6 \mathrm{H}\right), 1.56\left(\mathrm{~s}, \mathrm{CH}_{3}, 3 \mathrm{H}\right), 1.51\left(\mathrm{~s}, \mathrm{CH}_{3}, 6 \mathrm{H}\right), 1.42\left(\mathrm{~s}, \mathrm{CH}_{3}, 6 \mathrm{H}\right)$, $1.31\left(\mathrm{~s}, \mathrm{CH}_{3}, 3 \mathrm{H}\right) .{ }^{1} \mathrm{H}$ NMR $\left(500 \mathrm{MHz},-60{ }^{\circ} \mathrm{C}\right.$, THF- $\left.d 8\right): \delta 7.65\left(\mathrm{~d}, J=5.0 \mathrm{~Hz}, \mathrm{H}_{\mathrm{pyr}}, 2 \mathrm{H}\right), 6.83$ (d, $\left.J=4.8 \mathrm{~Hz}, \mathrm{H}_{\mathrm{pyr}}, 2 \mathrm{H}\right), 5.66\left(\mathrm{~d}, J=2.7 \mathrm{~Hz}, \mathrm{H}_{\mathrm{pyr}}, 2 \mathrm{H}\right), 5.61\left(\mathrm{~d}, J=2.6 \mathrm{~Hz}, \mathrm{H}_{\mathrm{pyr}}, 2 \mathrm{H}\right), 1.67$ (s, $\left.\mathrm{CH}_{3}, 3 \mathrm{H}\right), 1.63\left(\mathrm{~s}, \mathrm{CH}_{3}, 3 \mathrm{H}\right), 1.54\left(\mathrm{~s}, \mathrm{CH}_{3}, 2 \mathrm{H}\right), 1.51\left(\mathrm{~s}, \mathrm{CH}_{3}, 6 \mathrm{H}\right), 1.42\left(\mathrm{~s}, \mathrm{CH}_{3}, 6 \mathrm{H}\right), 1.33$ (s, 
$\left.\mathrm{CH}_{3}, 3 \mathrm{H}\right) .{ }^{1} \mathrm{H}$ NMR $\left(500 \mathrm{MHz},-80{ }^{\circ} \mathrm{C}, \mathrm{THF}-d_{8}\right): \delta 7.65\left(\mathrm{~d}, J=4.9 \mathrm{~Hz}, \mathrm{H}_{\mathrm{pyr}}, 2 \mathrm{H}\right), 6.82(\mathrm{~d}, J=4.9$ $\left.\mathrm{Hz}, \mathrm{H}_{\text {pyr }}, 2 \mathrm{H}\right), 5.65$ (d, $\left.J=2.5 \mathrm{~Hz}, \mathrm{H}_{\text {pyr }}, 2 \mathrm{H}\right), 5.60$ (d, $\left.J=2.1 \mathrm{~Hz}, \mathrm{H}_{\text {pyr }}, 2 \mathrm{H}\right), 1.70\left(\mathrm{~s}, \mathrm{CH}_{3}, 3 \mathrm{H}\right)$, $1.64\left(\mathrm{~s}, \mathrm{CH}_{3}, 3 \mathrm{H}\right), 1.52$ (s, $\left.\mathrm{CH}_{3}, 3 \mathrm{H}\right), 1.50\left(\mathrm{~s}, \mathrm{CH}_{3}, 6 \mathrm{H}\right), 1.41$ (s, $\left.\mathrm{CH}_{3}, 6 \mathrm{H}\right), 1.36\left(\mathrm{~s}, \mathrm{CH}_{3}, 3 \mathrm{H}\right) .{ }^{7} \mathrm{Li}$ NMR (155 MHz, $25{ }^{\circ} \mathrm{C}$, THF- $\left.d 8\right): \delta 0.79$ (s, 1Li), -1.09 (s, $\left.1 \mathrm{Li}\right) .{ }^{13} \mathrm{C}\left\{{ }^{1} \mathrm{H}\right\}$ NMR $(126 \mathrm{MHz},-30$ $\left.{ }^{\circ} \mathrm{C}, \mathrm{THF}-d_{8}\right): \delta 187.59$ (6), 150.88 (5/4), $150.06(\mathbf{5} / \mathbf{4}), 144.17$ (12), 128.35 (11), 101.75 (10), 99.00 (9), 87.53 (3), 68.39 (THF), 47.50 (13), 41.81 (7), 39.26 (2), 37.27 (14), 31.53 (1), 30.61 (8), 30.23 (8), 26.58 (THF). IR (KBr pellet, $\mathrm{cm}^{-1}$ ): 3089 (m), 3076 (m), 2962 (s), 2926 (s), 2866 (s), 1576, (s), 1460, (s), 1367 (m), 1350 (s), 1277 (m), 1244 (w), 1203 (m), 1155 (m), 1126 (w), 1092 (m), 1047 (s), 1022 (m), 916 (m), 895 (m), 800 (s), 802 (s), 741 (w), 725 (s), 609 (m), 577 (w), 557 (vw), 536 (vw), 499 (vw), 445 (m), 436 (m), 417 (s).

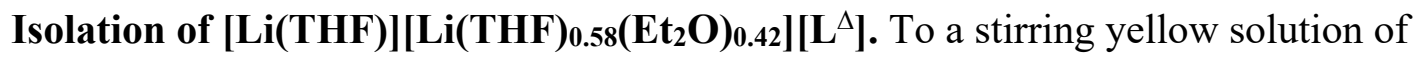
$\left[\mathrm{UO}_{2} \mathrm{Cl}_{2}(\mathrm{THF})_{2}\right]_{2}(69.9 \mathrm{mg}, 0.0717 \mathrm{mmol})$ in THF $(2 \mathrm{~mL})$ was added slowly a THF solution (2 $\mathrm{mL})$ of $[\mathrm{Li}(\mathrm{THF})]_{4}[\mathrm{~L}](106.3 \mathrm{mg}, 0.143 \mathrm{mmol})$. This resulted in a rapid color change to deep brown. The mixture was allowed to stir for $30 \mathrm{~min}$ before the volatiles were removed in vacuo, which afforded a brown-orange solid. The brown-orange residue was extracted into $\mathrm{Et}_{2} \mathrm{O}(3 \times 3$ $\mathrm{mL})$ and filtered through a Celite column supported on glass wool $(0.5 \mathrm{~cm} \times 2 \mathrm{~cm})$. This yielded a brown-orange filtrate and left a brown-black solid on the Celite column. The volume of this solution was reduced in vacuo to $5 \mathrm{~mL}$. Storage of this solution for $24 \mathrm{~h}$ at $-25^{\circ} \mathrm{C}$ resulted in the deposition of X-ray quality orange blocks of $[\mathrm{Li}(\mathrm{THF})]\left[\mathrm{Li}(\mathrm{THF})_{0.58}\left(\mathrm{Et}_{2} \mathrm{O}\right)_{0.42}\right]\left[\mathrm{L}^{\Delta}\right]$, which were isolated by decanting off the supernatant and drying in vacuo. Yield $33.4 \mathrm{mg}, 40 \%$. ${ }^{1} \mathrm{H}$ NMR (500 MHz, $\left.25{ }^{\circ} \mathrm{C}, \mathrm{THF}-d 8\right) \delta 7.59$ (br s, $\left.\mathrm{H}_{\mathrm{pyr}}, 2 \mathrm{H}\right), 6.83$ (br s, $\left.\mathrm{H}_{\mathrm{pyr}}, 2 \mathrm{H}\right), 5.67$ (br s, $\mathrm{H}_{\mathrm{pyr}}, 4 \mathrm{H}$ ), 3.54 (s, THF, 4H), 3.35 (q, J=7.0 Hz, Et2 $\mathrm{O}, 4 \mathrm{H}$ ), 1.69 (s, THF, 4H), 1.54 (s, $\left.\mathrm{CH}_{3}, 12 \mathrm{H}\right), 1.50$ (s, $\left.\mathrm{CH}_{3}, 3 \mathrm{H}\right), 1.42\left(\mathrm{~s}, \mathrm{CH}_{3}, 6 \mathrm{H}\right) 1.26\left(\mathrm{~s}, \mathrm{CH}_{3}, 3 \mathrm{H}\right), 1.08\left(\mathrm{t}, J=7.0 \mathrm{~Hz}, \mathrm{Et}_{2} \mathrm{O}, 6 \mathrm{H}\right)$.

Synthesis of [ $\mathbf{L i}(\mathbf{T H F})]\left[\mathbf{U O}_{2}\left(\mathbf{L}^{\Delta}\right) \mathbf{C l}(\mathbf{T H F})\right]$ (2). To a stirring, $-25{ }^{\circ} \mathrm{C}$ solution of $\left[\mathrm{UO}_{2} \mathrm{Cl}_{2}(\mathrm{THF})_{2}\right]_{2}(90.1 \mathrm{mg}, 0.0929 \mathrm{mmol})$ in THF $(2 \mathrm{~mL})$ was added dropwise a $-25^{\circ} \mathrm{C}$ solution of $1(108.2 \mathrm{mg}, 0.186 \mathrm{mmol})$ in THF $(2 \mathrm{~mL})$. This addition resulted in an immediate color change from yellow to deep green. As soon as the addition was complete, the volatiles were removed in vacuo and the resulting solids were triturated with pentane $(2 \times 2 \mathrm{~mL})$, affording a deep green solid. This solid was then extracted into toluene $(6 \mathrm{~mL})$ and filtered through a Celite column supported on glass wool $(0.5 \mathrm{~cm} \times 2 \mathrm{~cm})$. This yielded a deep green solution. The 
volume of this solution was reduced in vacuo to $3 \mathrm{~mL}$, filtered through a Celite column supported on glass wool $(0.5 \mathrm{~cm} \times 2 \mathrm{~cm})$, and layered with hexanes $(8 \mathrm{~mL})$. Storage of the solution at $-25{ }^{\circ} \mathrm{C}$ for $48 \mathrm{~h}$ resulted in the deposition of deep green needles of [Li(THF)][ $\left[\mathrm{UO}_{2}\left(\mathrm{~L}^{\Delta}\right) \mathrm{Cl}(\mathrm{THF})\right]$ (2), which were isolated by decanting off the supernatant (yield 80 mg, 49\%). Anal. Calcd for $\mathrm{C}_{36} \mathrm{H}_{48} \mathrm{~N}_{4} \mathrm{LiClO}_{4} \mathrm{U}$ : C, 49.07; H, 5.49; N, 6.36. Found: C, 49.44; H, 5.63; N, 6.12. ${ }^{1} \mathrm{H}$ NMR $\left(500 \mathrm{MHz}, 25^{\circ} \mathrm{C}, \mathrm{THF}-d_{8}\right): \delta 7.50-6.00$ (br m, $\left.\mathrm{H}_{\mathrm{pyr}}, 8 \mathrm{H}\right), 1.92$ (br s, $\left.\mathrm{CH}_{3}, 6 \mathrm{H}\right), 1.86$ (br s, $\left.\mathrm{CH}_{3}, 3 \mathrm{H}\right), 1.69$ (br s, $\left.\mathrm{CH}_{3}, 3 \mathrm{H}\right), 1.65$ (br s, $\left.\mathrm{CH}_{3}, 6 \mathrm{H}\right), 1.46\left(\mathrm{~s}, \mathrm{CH}_{3}, 3 \mathrm{H}\right.$ ), $1.39\left(\mathrm{~s}, \mathrm{CH}_{3}, 3 \mathrm{H}\right) .{ }^{1} \mathrm{H}$ NMR $\left(500 \mathrm{MHz}, 10^{\circ} \mathrm{C}\right.$, THF- $\left.d 8\right): \delta 7.50-5.93\left(\mathrm{br} \mathrm{m}, \mathrm{H}_{\mathrm{pyr}}, 8 \mathrm{H}\right), 1.94(\mathrm{br}$ s, $\left.\mathrm{CH}_{3}, 6 \mathrm{H}\right), 1.85$ (s, $\left.\mathrm{CH}_{3}, 3 \mathrm{H}\right), 1.69$ (br s, $\left.\mathrm{CH}_{3}, 3 \mathrm{H}\right), 1.64$ (br s, $\left.\mathrm{CH}_{3}, 6 \mathrm{H}\right), 1.47$ (s, $\mathrm{CH}_{3}, 3 \mathrm{H}$ ), 1.39 (s, $\left.\mathrm{CH}_{3}, 3 \mathrm{H}\right) .{ }^{1} \mathrm{H}$ NMR $\left(500 \mathrm{MHz}, 0{ }^{\circ} \mathrm{C}\right.$, THF- $d 8$ ): $\delta 7.17$ (br s, $\mathrm{H}_{\mathrm{pyr}}, 1 \mathrm{H}$ ), 6.95 (br s, $\mathrm{H}_{\mathrm{pyr}}$, 1H), 6.89 (br s, $\left.\mathrm{H}_{\mathrm{pyr}}, 1 \mathrm{H}\right), 6.74$ (br s, $\mathrm{H}_{\mathrm{pyr}}, 1 \mathrm{H}$ ), 6.56 (br s, $\mathrm{H}_{\mathrm{pyr}}, 1 \mathrm{H}$ ), 6.30 (br s, $\mathrm{H}_{\mathrm{pyr}}, 2 \mathrm{H}$ ), 6.04 (br s, $\mathrm{H}_{\text {pyr }}, 1 \mathrm{H}$ ), 2.01 (br s, $\left.\mathrm{CH}_{3}, 3 \mathrm{H}\right), 1.85\left(\mathrm{~s}, \mathrm{CH}_{3}, 6 \mathrm{H}\right), 1.69$ (s, $\left.\mathrm{CH}_{3}, 3 \mathrm{H}\right), 1.64$ (br s, $\mathrm{CH}_{3}, 6 \mathrm{H}$ ), 1.47 (s, $\left.\mathrm{CH}_{3}, 3 \mathrm{H}\right), 1.39$ (s, $\left.\mathrm{CH}_{3}, 3 \mathrm{H}\right) .{ }^{1} \mathrm{H}$ NMR $\left(500 \mathrm{MHz},-10{ }^{\circ} \mathrm{C}, \mathrm{THF}-d_{8}\right): \delta 7.21\left(\mathrm{~s}, \mathrm{H}_{\mathrm{pyr}}, 1 \mathrm{H}\right)$, 6.95 (s, $\left.\mathrm{H}_{\mathrm{pyr}}, 1 \mathrm{H}\right), 6.90$ (s, Hpyr, 1H), 6.76 (s, $\left.\mathrm{H}_{\mathrm{pyr}}, 1 \mathrm{H}\right), 6.57$ (s, $\left.\mathrm{H}_{\mathrm{pyr}}, 1 \mathrm{H}\right), 6.30$ (s, $\left.\mathrm{H}_{\mathrm{pyr}}, 1 \mathrm{H}\right), 6.26$ (s, $\left.\mathrm{H}_{\text {pyr }}, 1 \mathrm{H}\right), 6.04$ (s, $\left.\mathrm{H}_{\text {pyr }}, 1 \mathrm{H}\right), 2.02$ (br s, $\left.\mathrm{CH}_{3}, 3 \mathrm{H}\right), 1.84$ (s, $\left.\mathrm{CH}_{3}, 6 \mathrm{H}\right), 1.70\left(\mathrm{~s}, \mathrm{CH}_{3}, 3 \mathrm{H}\right), 1.66$ (br s, $\left.\mathrm{CH}_{3}, 3 \mathrm{H}\right), 1.62$ (br s, $\left.\mathrm{CH}_{3}, 3 \mathrm{H}\right), 1.47$ (s, $\left.\mathrm{CH}_{3}, 3 \mathrm{H}\right), 1.38$ (s, $\left.\mathrm{CH}_{3}, 3 \mathrm{H}\right) .{ }^{1} \mathrm{H}$ NMR (500 MHz, $\left.20{ }^{\circ} \mathrm{C}, \mathrm{THF}-d_{8}\right): \delta 7.22\left(\mathrm{~d}, J=4.9 \mathrm{~Hz}, \mathrm{H}_{\mathrm{pyr}}, 1 \mathrm{H}\right), 6.97\left(\mathrm{~d}, J=4.7 \mathrm{~Hz}, \mathrm{H}_{\mathrm{pyr}}, 1 \mathrm{H}\right), 6.91\left(\mathrm{~s}, \mathrm{H}_{\mathrm{pyr}}, 1 \mathrm{H}\right)$, 6.78 (d, $\left.J=4.9 \mathrm{~Hz}, \mathrm{H}_{\mathrm{pyr}}, 1 \mathrm{H}\right), 6.58$ (s, $\left.\mathrm{H}_{\mathrm{pyr}}, 1 \mathrm{H}\right), 6.30$ (d, $\left.J=3.3 \mathrm{~Hz}, \mathrm{H}_{\mathrm{pyr}}, 1 \mathrm{H}\right), 6.26$ (d, $J=4.8$ $\left.\mathrm{Hz}, \mathrm{H}_{\mathrm{pyr}}, 1 \mathrm{H}\right), 6.04$ (d, J=3.1 Hz, $\left.\mathrm{H}_{\mathrm{pyr}}, 1 \mathrm{H}\right), 2.03$ (s, $\left.\mathrm{CH}_{3}, 3 \mathrm{H}\right), 1.83$ (s, $\left.\mathrm{CH}_{3}, 6 \mathrm{H}\right), 1.70$ (s, $\mathrm{CH}_{3}$, $3 \mathrm{H}), 1.66$ (s, $\left.\mathrm{CH}_{3}, 3 \mathrm{H}\right), 1.61$ (s, $\left.\mathrm{CH}_{3}, 3 \mathrm{H}\right), 1.47$ (s, $\left.\mathrm{CH}_{3}, 3 \mathrm{H}\right), 1.38\left(\mathrm{~s}, \mathrm{CH}_{3}, 3 \mathrm{H}\right) .{ }^{1} \mathrm{H}$ NMR (500 MHz, $\left.-30{ }^{\circ} \mathrm{C}, \mathrm{THF}-d_{8}\right): \delta 7.24\left(\mathrm{~d}, J=5.0 \mathrm{~Hz}, \mathrm{H}_{\mathrm{pyr}}, 1 \mathrm{H}\right), 6.98\left(\mathrm{~d}, J=4.9 \mathrm{~Hz}, \mathrm{H}_{\mathrm{pyr}}, 1 \mathrm{H}\right), 6.91(\mathrm{~d}, J$ $\left.=2.8 \mathrm{~Hz}, \mathrm{H}_{\mathrm{pyr}}, 1 \mathrm{H}\right), 6.80\left(\mathrm{~d}, J=4.9 \mathrm{~Hz}, \mathrm{H}_{\mathrm{pyr}}, 1 \mathrm{H}\right), 6.59\left(\mathrm{~d}, J=2.8 \mathrm{~Hz}, \mathrm{H}_{\mathrm{pyr}}, 1 \mathrm{H}\right), 6.30(\mathrm{~d}, J=3.0$ $\left.\mathrm{Hz}, \mathrm{H}_{\mathrm{pyr}}, 1 \mathrm{H}\right), 6.25$ (d, J=4.9 Hz, Hpyr, $\left.1 \mathrm{H}\right), 6.03$ (d, $\left.J=3.1 \mathrm{~Hz}, \mathrm{H}_{\mathrm{pyr}}, 1 \mathrm{H}\right), 2.03$ (s, $\left.\mathrm{CH}_{3}, 3 \mathrm{H}\right)$, 1.82 (s, $\left.\mathrm{CH}_{3}, 6 \mathrm{H}\right), 1.69$ (s, $\left.\mathrm{CH}_{3}, 3 \mathrm{H}\right), 1.66\left(\mathrm{~s}, \mathrm{CH}_{3}, 3 \mathrm{H}\right), 1.61$ (s, $\left.\mathrm{CH}_{3}, 3 \mathrm{H}\right), 1.48\left(\mathrm{~s}, \mathrm{CH}_{3}, 3 \mathrm{H}\right)$, 1.38 (s, $\left.\mathrm{CH}_{3}, 3 \mathrm{H}\right) .{ }^{1} \mathrm{H}$ NMR (500 MHz, $-40{ }^{\circ} \mathrm{C}$, THF- $\left.d 8\right): \delta 7.25$ (d, $\left.J=4.8 \mathrm{~Hz}, \mathrm{H}_{\mathrm{pyr}}, 1 \mathrm{H}\right), 6.99$ (d, $\left.J=4.7 \mathrm{~Hz}, \mathrm{H}_{\mathrm{pyr}}, 1 \mathrm{H}\right), 6.92\left(\mathrm{~d}, J=2.8 \mathrm{~Hz}, \mathrm{H}_{\mathrm{pyr}}, 1 \mathrm{H}\right), 6.82\left(\mathrm{~d}, J=4.7 \mathrm{~Hz}, \mathrm{H}_{\mathrm{pyr}}, 1 \mathrm{H}\right), 6.59$ (d, $J$ $\left.=2.8 \mathrm{~Hz}, \mathrm{H}_{\mathrm{pyr}}, 1 \mathrm{H}\right), 6.30\left(\mathrm{~d}, J=3.1 \mathrm{~Hz}, \mathrm{H}_{\mathrm{pyr}}, 1 \mathrm{H}\right), 6.26\left(\mathrm{~d}, J=4.9 \mathrm{~Hz}, \mathrm{H}_{\mathrm{pyr}}, 1 \mathrm{H}\right), 6.03(\mathrm{~d}, J=3.1$ $\left.\mathrm{Hz}, \mathrm{H}_{\text {pyr }}, 1 \mathrm{H}\right), 2.03$ (s, $\left.\mathrm{CH}_{3}, 3 \mathrm{H}\right), 1.82$ (s, $\left.\mathrm{CH}_{3}, 6 \mathrm{H}\right), 1.69$ (s, $\left.\mathrm{CH}_{3}, 3 \mathrm{H}\right), 1.66$ (s, $\left.\mathrm{CH}_{3}, 3 \mathrm{H}\right), 1.61$ (s, $\left.\mathrm{CH}_{3}, 3 \mathrm{H}\right), 1.48\left(\mathrm{~s}, \mathrm{CH}_{3}, 3 \mathrm{H}\right), 1.38\left(\mathrm{~s}, \mathrm{CH}_{3}, 3 \mathrm{H}\right) .{ }^{1} \mathrm{H}$ NMR $\left(500 \mathrm{MHz},-50{ }^{\circ} \mathrm{C}\right.$, THF- $\left.d_{8}\right): \delta 7.27(\mathrm{~d}$, $\left.J=4.9 \mathrm{~Hz}, \mathrm{H}_{\mathrm{pyr}}, 1 \mathrm{H}\right), 7.01$ (d, $\left.J=4.9 \mathrm{~Hz}, \mathrm{H}_{\mathrm{pyr}}, 1 \mathrm{H}\right), 6.92\left(\mathrm{~d}, J=2.8 \mathrm{~Hz}, \mathrm{H}_{\mathrm{pyr}}, 1 \mathrm{H}\right), 6.84$ (d, $J=$ $\left.4.9 \mathrm{~Hz}, \mathrm{H}_{\mathrm{pyr}}, 1 \mathrm{H}\right), 6.60$ (d, $\left.J=2.8 \mathrm{~Hz}, \mathrm{H}_{\mathrm{pyr}}, 1 \mathrm{H}\right), 6.30$ (d, $\left.J=3.1 \mathrm{~Hz}, \mathrm{H}_{\text {pyr }}, 1 \mathrm{H}\right), 6.26$ (d, $J=4.9$ 
$\left.\mathrm{Hz}, \mathrm{H}_{\mathrm{pyr}}, 1 \mathrm{H}\right), 6.03$ (d, J=3.1 Hz, Hpyr $\left.1 \mathrm{H}\right), 2.04$ (s, $\left.\mathrm{CH}_{3}, 3 \mathrm{H}\right), 1.82\left(\mathrm{~s}, \mathrm{CH}_{3}, 6 \mathrm{H}\right), 1.68\left(\mathrm{~s}, \mathrm{CH}_{3}\right.$, $3 \mathrm{H}), 1.66\left(\mathrm{~s}, \mathrm{CH}_{3}, 3 \mathrm{H}\right), 1.60\left(\mathrm{~s}, \mathrm{CH}_{3}, 3 \mathrm{H}\right), 1.48\left(\mathrm{~s}, \mathrm{CH}_{3}, 3 \mathrm{H}\right), 1.37\left(\mathrm{~s}, \mathrm{CH}_{3}, 3 \mathrm{H}\right) .{ }^{13} \mathrm{C}\left\{{ }^{1} \mathrm{H}\right\} \mathrm{NMR}$ (126 MHz, $\left.-30{ }^{\circ} \mathrm{C}, \mathrm{THF}-d 8\right): \delta 187.77,187.14,166.01,163.74,152.40,151.72,149.91,146.21$, 130.65, 127.67, 127.05, 118.16, 108.73, 108.56, 105.73, 88.25, 47.00, 42.70, 40.69, 40.01, 39.84, 39.54, 34.26, 30.85, 30.70. ${ }^{7} \mathrm{Li}\left\{{ }^{1} \mathrm{H}\right\} \mathrm{NMR}\left(155 \mathrm{MHz}, 25{ }^{\circ} \mathrm{C}\right.$, THF- $\left.d_{8}\right): \delta-0.18$ (s). IR (KBr pellet, $\mathrm{cm}^{-1}$ ): 3097 (w), 3080 (w), 2962 (s), 2920 (s), 2875 (m), 1570 (s), 1493 (w), 1464 (m), $1381(\mathrm{w}), 1362(\mathrm{w}), 1352(\mathrm{~m}), 1265(\mathrm{~m}), 1244(\mathrm{w}), 1207$ (m), $1178(\mathrm{w}), 1155(\mathrm{~m}), 1134(\mathrm{w})$, $1113(\mathrm{w}), 1101$ (m), 1051 (s), 1034 (w), 1011 (w), 984 (w), 974 (w), $891(\mathrm{vs}), 858$ (s), 810 (s), $795(\mathrm{~s}), 752(\mathrm{~m}), 731(\mathrm{~m}), 696(\mathrm{w}), 673(\mathrm{w}), 606(\mathrm{~m}), 501(\mathrm{w})$.

Synthesis of [Li(THF)][U $\left.\mathbf{O}_{2}\left(\mathbf{L}^{\Delta}\right)(\mathbf{O T f})(\mathrm{THF})\right](3)$. To a stirring $-25{ }^{\circ} \mathrm{C}$ solution of $\left[\mathrm{UO}_{2}(\mathrm{OTf})_{2}(\mathrm{THF})_{3}\right](121.2 \mathrm{mg}, 0.155 \mathrm{mmol})$ in THF $(2 \mathrm{~mL})$ was added dropwise a $-25^{\circ} \mathrm{C}$ solution of $1(114.8 \mathrm{mg}, 0.155 \mathrm{mmol})$ in THF $(2 \mathrm{~mL})$. This addition resulted in an immediate color change from yellow to deep green. As soon as the addition was complete, the deep greenbrown solution was filtered through a Celite column supported on glass wool $(0.5 \mathrm{~cm} \times 2 \mathrm{~cm})$. This yielded a deep green-brown solution. Layering of this solution with hexanes $(8 \mathrm{~mL})$ followed storage of the solution at $-25{ }^{\circ} \mathrm{C}$ for $24 \mathrm{~h}$ resulted in the deposition of copious amounts of a brown solid, which we have tentatively ascribed to $\mathrm{L}^{\Delta \Delta}$ (Figure S14). Filtration of this solution and further storage at $-25^{\circ} \mathrm{C}$ for $24 \mathrm{~h}$ resulted in the deposition of deep green needles of $[\mathrm{Li}(\mathrm{THF})]\left[\mathrm{UO}_{2}\left(\mathrm{~L}^{\Delta}\right)(\mathrm{OTf})(\mathrm{THF})\right](3)$, which were isolated by decanting off the supernatant (yield $35.1 \mathrm{mg}, 30 \%$ ). Anal. Calcd for $\mathrm{C}_{37} \mathrm{H}_{48} \mathrm{~F}_{3} \mathrm{LiN}_{4} \mathrm{O}_{7} \mathrm{SU}$ : C, 44.67; H, 4.86; N, 5.63. Found: C, 44.13; $\mathrm{H}, 4.58 ; \mathrm{N}, 5.27 .{ }^{1} \mathrm{H}$ NMR $\left(500 \mathrm{MHz}, 25{ }^{\circ} \mathrm{C}, \mathrm{THF}-d_{8}\right): \delta 7.32-5.87$ (br m, 8H), $1.98(\mathrm{~s}, 3 \mathrm{H})$, 1.83 (br s, 6H), 1.73 (s, 6H), 1.68 (s, 3H), 1.48 (s, 3H), $1.42(\mathrm{~s}, 3 \mathrm{H}) .{ }^{1} \mathrm{H}$ NMR $\left(500 \mathrm{MHz}, 10{ }^{\circ} \mathrm{C}\right.$, THF-ds): $\delta 7.19$ (br s, $\left.\mathrm{H}_{\text {pyr }}, 1 \mathrm{H}\right), 6.95$ (br s, $\left.\mathrm{H}_{\text {pyr }}, 1 \mathrm{H}\right), 6.85$ (br s, $\left.\mathrm{H}_{\text {pyr }}, 1 \mathrm{H}\right), 6.72$ (br s, $\mathrm{H}_{\text {pyr }}, 1 \mathrm{H}$ ), 6.63 (br s, $\mathrm{H}_{\text {pyr }}, 1 \mathrm{H}$ ), 6.22 (br s, $\left.\mathrm{H}_{\text {pyr }}, 2 \mathrm{H}\right), 6.05$ (br s, $\left.\mathrm{H}_{\text {pyr, }} 1 \mathrm{H}\right), 1.97$ (s, $\left.\mathrm{CH}_{3}, 6 \mathrm{H}\right), 1.74$ (s, $\mathrm{CH}_{3}$, 3H) $1.72\left(\mathrm{~s}, \mathrm{CH}_{3}, 6 \mathrm{H}\right), 1.64$ (br s, $\left.\mathrm{CH}_{3}, 3 \mathrm{H}\right), 1.48\left(\mathrm{~s}, \mathrm{CH}_{3}, 3 \mathrm{H}\right), 1.41\left(\mathrm{~s}, \mathrm{CH}_{3}, 3 \mathrm{H}\right) .{ }^{1} \mathrm{H} \mathrm{NMR}(500$ $\left.\mathrm{MHz}, 0^{\circ} \mathrm{C}, \mathrm{THF}-d_{8}\right): \delta 7.21$ (s, $\left.\mathrm{H}_{\text {pyr }}, 1 \mathrm{H}\right), 6.96$ (s, $\left.\mathrm{H}_{\text {pyr }}, 1 \mathrm{H}\right), 6.86$ (s, $\left.\mathrm{H}_{\text {pyr }}, 1 \mathrm{H}\right), 6.75$ (s, $\left.\mathrm{H}_{\text {pyr }}, 1 \mathrm{H}\right)$, 6.64 (s, Hpyr, 1H), 6.23 (s, Hyr, 1H), 6.21 (s, Hyr, 1H), 6.05 (s, H $\left.\mathrm{H}_{\text {pyr }}, 1 \mathrm{H}\right), 1.97$ (s, $\left.\mathrm{CH}_{3}, 6 \mathrm{H}\right), 1.74$ $\left(\mathrm{s}, \mathrm{CH}_{3}, 3 \mathrm{H}\right), 1.73\left(\mathrm{~s}, \mathrm{CH}_{3}, 6 \mathrm{H}\right), 1.63\left(\mathrm{~s}, \mathrm{CH}_{3}, 3 \mathrm{H}\right), 1.48\left(\mathrm{~s}, \mathrm{CH}_{3}, 3 \mathrm{H}\right), 1.41\left(\mathrm{~s}, \mathrm{CH}_{3}, 3 \mathrm{H}\right) .{ }^{1} \mathrm{H}$ NMR (500 MHz, $-10^{\circ} \mathrm{C}$ THF-d8): $\delta 7.22\left(\mathrm{~d}, J=4.9 \mathrm{~Hz}, \mathrm{H}_{\text {pyr }}, 1 \mathrm{H}\right), 6.97$ (d, J=4.9 Hz, H $6.87\left(\mathrm{~s}, \mathrm{H}_{\mathrm{pyr}}, 1 \mathrm{H}\right), 6.76\left(\mathrm{~d}, J=4.9 \mathrm{~Hz}, \mathrm{H}_{\mathrm{pyr}}, 1 \mathrm{H}\right), 6.65\left(\mathrm{~s}, \mathrm{H}_{\mathrm{pyr}}, 1 \mathrm{H}\right), 6.24\left(\mathrm{~s}, \mathrm{H}_{\mathrm{pyr}}, 1 \mathrm{H}\right), 6.20(\mathrm{~d}, J=$ $\left.4.8 \mathrm{~Hz}, \mathrm{H}_{\text {pyr }}, 1 \mathrm{H}\right), 6.04$ (s, $\left.\mathrm{H}_{\text {pyr, }} 1 \mathrm{H}\right), 1.97$ (s, $\left.\mathrm{CH}_{3}, 3 \mathrm{H}\right), 1.96\left(\mathrm{~s}, \mathrm{CH}_{3}, 3 \mathrm{H}\right), 1.73\left(\mathrm{~s}, \mathrm{CH}_{3}, 3 \mathrm{H}\right), 1.72$ 
(s, $\left.\mathrm{CH}_{3}, 6 \mathrm{H}\right), 1.63\left(\mathrm{~s}, \mathrm{CH}_{3}, 3 \mathrm{H}\right), 1.48\left(\mathrm{~s}, \mathrm{CH}_{3}, 3 \mathrm{H}\right), 1.41$ (s, $\left.\mathrm{CH}_{3}, 3 \mathrm{H}\right) .{ }^{1} \mathrm{H}$ NMR (500 MHz, -20 $\left.{ }^{\circ} \mathrm{C}, \mathrm{THF}-d 8\right): \delta 7.23$ (d, $\left.J=5.0 \mathrm{~Hz}, \mathrm{H}_{\mathrm{pyr}}, 1 \mathrm{H}\right), 6.98\left(\mathrm{~d}, J=5.0 \mathrm{~Hz}, \mathrm{H}_{\mathrm{pyr}}, 1 \mathrm{H}\right), 6.87$ (d, $J=2.8 \mathrm{~Hz}$, $\left.\mathrm{H}_{\mathrm{pyr}}, 1 \mathrm{H}\right), 6.78$ (d, $\left.J=5.0 \mathrm{~Hz}, \mathrm{H}_{\mathrm{pyr}}, 1 \mathrm{H}\right), 6.65$ (d, $\left.J=3.1 \mathrm{~Hz}, \mathrm{H}_{\mathrm{pyr}}, 1 \mathrm{H}\right), 6.24$ (d, $J=3.1 \mathrm{~Hz}, \mathrm{H}_{\mathrm{pyr}}$, 1H), 6.20 (d, $\left.J=5.0 \mathrm{~Hz}, \mathrm{H}_{\mathrm{pyr}}, 1 \mathrm{H}\right), 6.04$ (d, $\left.J=3.1 \mathrm{~Hz}, \mathrm{H}_{\mathrm{pyr}}, 1 \mathrm{H}\right), 1.98$ (s, $\left.\mathrm{CH}_{3}, 3 \mathrm{H}\right), 1.96$ (s, $\left.\mathrm{CH}_{3}, 3 \mathrm{H}\right), 1.75$ (s, $\left.\mathrm{CH}_{3}, 3 \mathrm{H}\right), 1.74\left(\mathrm{~s}, \mathrm{CH}_{3}, 3 \mathrm{H}\right), 1.72$ (s, $\left.\mathrm{CH}_{3}, 3 \mathrm{H}\right), 1.62\left(\mathrm{~s}, \mathrm{CH}_{3}, 3 \mathrm{H}\right), 1.49$ (s, $\left.\mathrm{CH}_{3}, 3 \mathrm{H}\right), 1.41$ (s, $\left.\mathrm{CH}_{3}, 3 \mathrm{H}\right) .{ }^{1} \mathrm{H}$ NMR $\left(500 \mathrm{MHz},-30^{\circ} \mathrm{C}\right.$, THF- $\left.d 8\right): \delta 7.24$ (d, $J=5.0 \mathrm{~Hz}, \mathrm{H}_{\text {pyr, }}$, $1 \mathrm{H}), 7.00$ (d, $\left.J=4.9 \mathrm{~Hz}, \mathrm{H}_{\mathrm{pyr}}, 1 \mathrm{H}\right), 6.88$ (d, $\left.J=2.8 \mathrm{~Hz}, \mathrm{H}_{\mathrm{pyr}}, 1 \mathrm{H}\right), 6.80$ (d, $\left.J=5.0 \mathrm{~Hz}, \mathrm{H}_{\mathrm{pyr}}, 1 \mathrm{H}\right)$, $6.67\left(\mathrm{~d}, J=2.7 \mathrm{~Hz}, \mathrm{H}_{\mathrm{pyr}}, 1 \mathrm{H}\right), 6.24\left(\mathrm{~d}, J=3.0 \mathrm{~Hz}, \mathrm{H}_{\mathrm{pyr}}, 1 \mathrm{H}\right), 6.20\left(\mathrm{~d}, J=5.0 \mathrm{~Hz}, \mathrm{H}_{\mathrm{pyr}}, 1 \mathrm{H}\right), 6.04$ (d, $\left.J=3.1 \mathrm{~Hz}, \mathrm{H}_{\text {pyr }}, 1 \mathrm{H}\right), 1.98$ (s, $\left.\mathrm{CH}_{3}, 3 \mathrm{H}\right), 1.95$ (s, $\left.\mathrm{CH}_{3}, 3 \mathrm{H}\right), 1.75$ (s, $\left.\mathrm{CH}_{3}, 3 \mathrm{H}\right), 1.74$ (s, $\mathrm{CH}_{3}$, $3 \mathrm{H}), 1.72\left(\mathrm{~s}, \mathrm{CH}_{3}, 3 \mathrm{H}\right), 1.62$ (s, $\left.\mathrm{CH}_{3}, 3 \mathrm{H}\right), 1.49$ (s, $\left.\mathrm{CH}_{3}, 3 \mathrm{H}\right), 1.41$ (s, $\left.\mathrm{CH}_{3}, 3 \mathrm{H}\right),{ }^{1} \mathrm{H}$ NMR (500 MHz, $\left.-40{ }^{\circ} \mathrm{C}, \mathrm{THF}-d_{8}\right): \delta 7.26$ (d, $\left.J=4.9 \mathrm{~Hz}, \mathrm{H}_{\mathrm{pyr}}, 1 \mathrm{H}\right), 7.02\left(\mathrm{~d}, J=4.9 \mathrm{~Hz}, \mathrm{H}_{\mathrm{pyr}}, 1 \mathrm{H}\right), 6.89$ (d, $J$ $\left.=2.8 \mathrm{~Hz}, \mathrm{H}_{\mathrm{pyr}}, 1 \mathrm{H}\right), 6.83\left(\mathrm{~d}, J=5.0 \mathrm{~Hz}, \mathrm{H}_{\mathrm{pyr}}, 1 \mathrm{H}\right), 6.69\left(\mathrm{~d}, J=2.8 \mathrm{~Hz}, \mathrm{H}_{\mathrm{pyr}}, 1 \mathrm{H}\right), 6.25(\mathrm{~d}, J=3.0$ $\left.\mathrm{Hz}, \mathrm{H}_{\mathrm{pyr}}, 1 \mathrm{H}\right), 6.21$ (d, J=5.0 Hz, Hpyr, 1H), 6.04 (d, $\left.J=3.0 \mathrm{~Hz}, \mathrm{H}_{\mathrm{pyr}}, 1 \mathrm{H}\right), 1.99\left(\mathrm{~s}, \mathrm{CH}_{3}, 3 \mathrm{H}\right)$, $1.94\left(\mathrm{~s}, \mathrm{CH}_{3}, 3 \mathrm{H}\right), 1.75$ (s, $\left.\mathrm{CH}_{3}, 3 \mathrm{H}\right), 1.74\left(\mathrm{~s}, \mathrm{CH}_{3}, 3 \mathrm{H}\right), 1.72\left(\mathrm{~s}, \mathrm{CH}_{3}, 3 \mathrm{H}\right), 1.61\left(\mathrm{~s}, \mathrm{CH}_{3}, 3 \mathrm{H}\right)$, $1.49\left(\mathrm{~s}, \mathrm{CH}_{3}, 3 \mathrm{H}\right), 1.40\left(\mathrm{~s}, \mathrm{CH}_{3}, 3 \mathrm{H}\right) .{ }^{1} \mathrm{H}$ NMR $\left(500 \mathrm{MHz},-50{ }^{\circ} \mathrm{C}\right.$, THF- $\left.d_{8}\right): \delta 7.27$ (d, $J=4.9$ $\left.\mathrm{Hz}, \mathrm{H}_{\mathrm{pyr}}, 1 \mathrm{H}\right), 7.03$ (d, $\left.J=5.0 \mathrm{~Hz}, \mathrm{H}_{\mathrm{pyr}}, 1 \mathrm{H}\right), 6.89$ (d, $\left.J=2.8 \mathrm{~Hz}, \mathrm{H}_{\mathrm{pyr}}, 1 \mathrm{H}\right), 6.85(\mathrm{~d}, J=5.0 \mathrm{~Hz}$, $\left.\mathrm{H}_{\mathrm{pyr}}, 1 \mathrm{H}\right), 6.70$ (d, $\left.J=2.8 \mathrm{~Hz}, \mathrm{H}_{\mathrm{pyr}}, 1 \mathrm{H}\right), 6.25$ (d, $\left.J=3.1 \mathrm{~Hz}, \mathrm{H}_{\mathrm{pyr}}, 1 \mathrm{H}\right), 6.21$ (d, $J=4.9 \mathrm{~Hz}, \mathrm{H}_{\mathrm{pyr}}$, 1H), 6.04 (d, $\left.J=3.0 \mathrm{~Hz}, \mathrm{H}_{\mathrm{pyr}}, 1 \mathrm{H}\right), 1.99$ (s, $\left.\mathrm{CH}_{3}, 3 \mathrm{H}\right), 1.93$ (s, $\left.\mathrm{CH}_{3}, 3 \mathrm{H}\right), 1.74\left(\mathrm{~s}, \mathrm{CH}_{3}, 3 \mathrm{H}\right), 1.73$ (s, $\left.\mathrm{CH}_{3}, 3 \mathrm{H}\right), 1.71\left(\mathrm{~s}, \mathrm{CH}_{3}, 3 \mathrm{H}\right), 1.61\left(\mathrm{~s}, \mathrm{CH}_{3}, 3 \mathrm{H}\right), 1.49\left(\mathrm{~s}, \mathrm{CH}_{3}, 3 \mathrm{H}\right), 1.40\left(\mathrm{~s}, \mathrm{CH}_{3}, 3 \mathrm{H}\right) .{ }^{7} \mathrm{Li}$ NMR (155 MHz, $25^{\circ} \mathrm{C}$, THF- $\left.d 8\right): \delta 0.02(\mathrm{~s}) .{ }^{19} \mathrm{~F}\left\{{ }^{1} \mathrm{H}\right\}$ NMR $\left(376 \mathrm{MHz}, 25{ }^{\circ} \mathrm{C}\right.$, THF- $\left.d 8\right): \delta$ -78.43. (s). IR (KBr pellet, $\mathrm{cm}^{-1}$ ): 3105 (w), 3080 (w), 2966 (s), 2927 (s), 2872 (m), 1572 (m), $1462(\mathrm{~m}), 1381(\mathrm{w}), 1338(\mathrm{w}), 1327$ (s), $1288(\mathrm{~m}), 1261(\mathrm{~m}), 1236(\mathrm{~s}), 1207$ (s), $1171(\mathrm{~m}), 1111$ (w), 1097 (w), $1028(\mathrm{~m}), 1018(\mathrm{~s}), 985(\mathrm{w}), 972(\mathrm{w}), 912(\mathrm{~s}), 854(\mathrm{~m}), 800(\mathrm{~m}), 783(\mathrm{~m}), 760(\mathrm{w})$, 733 (w), 634 (s), 604 (w), 571 (w), 515 (w), 498 (w), 451 (w), 426 (w).

Reversible Oxidation of $[\mathbf{L i}(\mathbf{T H F})]_{4}[\mathbf{L}]$. An NMR tube fitted with a J-Young valve was charged with $[\mathrm{Li}(\mathrm{THF})]_{4}[\mathrm{~L}](23.6 \mathrm{mg}, 0.031 \mathrm{mmol})$ and THF- $d_{8}(0.5 \mathrm{~mL})$. A ${ }^{1} \mathrm{H}$ NMR spectrum was recorded (Figure S16). ${ }^{1} \mathrm{H}$ NMR (400 MHz, $25^{\circ} \mathrm{C}$, THF- $\left.d_{8}\right): \delta 5.72\left(\mathrm{~s}, \mathrm{H}_{\mathrm{pyr}}, 8 \mathrm{H}\right), 1.46\left(\mathrm{~s}, \mathrm{CH}_{3}\right.$, 24H). The NMR tube was brought back inside the glovebox and a pale yellow slurry of $\left[\mathrm{UO}_{2} \mathrm{Cl}_{2}\left(\mathrm{Ph}_{3} \mathrm{PO}\right)_{2}\right](28.6 \mathrm{mg}, 0.031 \mathrm{mmol})$ in THF- $d_{8}(0.5 \mathrm{~mL})$ was added. The color of the solution quickly turned very deep-brown, but no precipitate was observed. A ${ }^{1} \mathrm{H}$ NMR spectrum was re-recorded, which revealed the presence of 1 and $\mathrm{H}_{4} \mathrm{~L}$ in a 10:2 ratio (Figure S16). ${ }^{1} \mathrm{H}$ NMR 
(400 MHz, $25^{\circ} \mathrm{C}$, THF- $d_{8}$ ): $\delta 7.53$ (br d, $\left.o-\mathrm{C}_{6} H_{5}, 6 \mathrm{H}\right) 7.47$ (t, $p-\mathrm{C}_{6} H_{5}, 3 \mathrm{H}$ ), $7.32\left(\mathrm{t}, m-\mathrm{C}_{6} H_{5}, 6 \mathrm{H}\right.$ ), 6.83 (br s, $\left.H_{\mathrm{pyr}} 1,2 \mathrm{H}\right), 5.67$ (br s, $\left.H_{\mathrm{pyr}}, 1,4 H\right), 5.63$ (s, $\left.H_{\mathrm{pyr}}, \mathrm{H}_{4} \mathrm{~L}, 8 \mathrm{H}\right), 1.79$ (s, $\left.\mathrm{H}_{4} \mathrm{~L}, \mathrm{CH}_{3}, 24 \mathrm{H}\right)$, 1.54 (br s, $\mathrm{CH}_{3}, 1$ 6H), 1.53 (br s, $\mathrm{CH}_{3}, \mathbf{1}, 9 \mathrm{H}$ ), 1.46 (br s, $\mathrm{CH}_{3}, \mathbf{1}, 6 \mathrm{H}$ ), 1.34 (br s, $\mathrm{CH}_{3}, \mathbf{1}, 3 \mathrm{H}$ ). The NMR tube was brought back inside the glovebox and $\mathrm{Li}^{0}$ metal was added to the tube as a silvery solid ( $0.6 \mathrm{mg}, 0.073 \mathrm{mmol}, 2.3$ equiv). The sample was agitated by shaking once per hour, over the course of $10 \mathrm{~h}$, and a ${ }^{1} \mathrm{H}$ NMR spectrum was re-recorded at $10 \mathrm{~h}$, which revealed the absence of 1 , along with the presence of $[\mathrm{Li}(\mathrm{THF})]_{4}[\mathrm{~L}]$ and $\mathrm{H}_{4} \mathrm{~L}$, in a 10:2.5 ratio. ${ }^{1} \mathrm{H}$ NMR $\left(400 \mathrm{MHz}, 25{ }^{\circ} \mathrm{C}\right.$, THF- $\left.d 8\right): \delta 7.53$ (br d, $\left.o-\mathrm{C}_{6} H_{5}, 6 \mathrm{H}\right) 7.47$ (t, $\left.p-\mathrm{C}_{6} H_{5}, 3 \mathrm{H}\right), 7.32\left(\mathrm{t}, m-\mathrm{C}_{6} H_{5}, 6 \mathrm{H}\right)$ $5.72\left(\mathrm{~s}, \mathrm{H}_{\mathrm{pyr}},[\mathrm{Li}(\mathrm{THF})]_{4}[\mathrm{~L}], 8 \mathrm{H}\right), 5.63\left(\mathrm{~s}, H_{\mathrm{pyr}}, \mathrm{H}_{4} \mathrm{~L}, 8 \mathrm{H}\right), 1.79$ (s, $\left.\mathrm{CH}_{3}, \mathrm{H}_{4} \mathrm{~L}, 24 \mathrm{H}\right), 1.46\left(\mathrm{~s}, \mathrm{CH}_{3}\right.$, [Li(THF) $\left.]_{4}[\mathrm{~L}], 24 \mathrm{H}\right)$. On standing for $24 \mathrm{~h}$, a deep-brown precipitate had formed on the walls of the NMR tube, consistent with formation of $\mathrm{UO}_{2}$. 


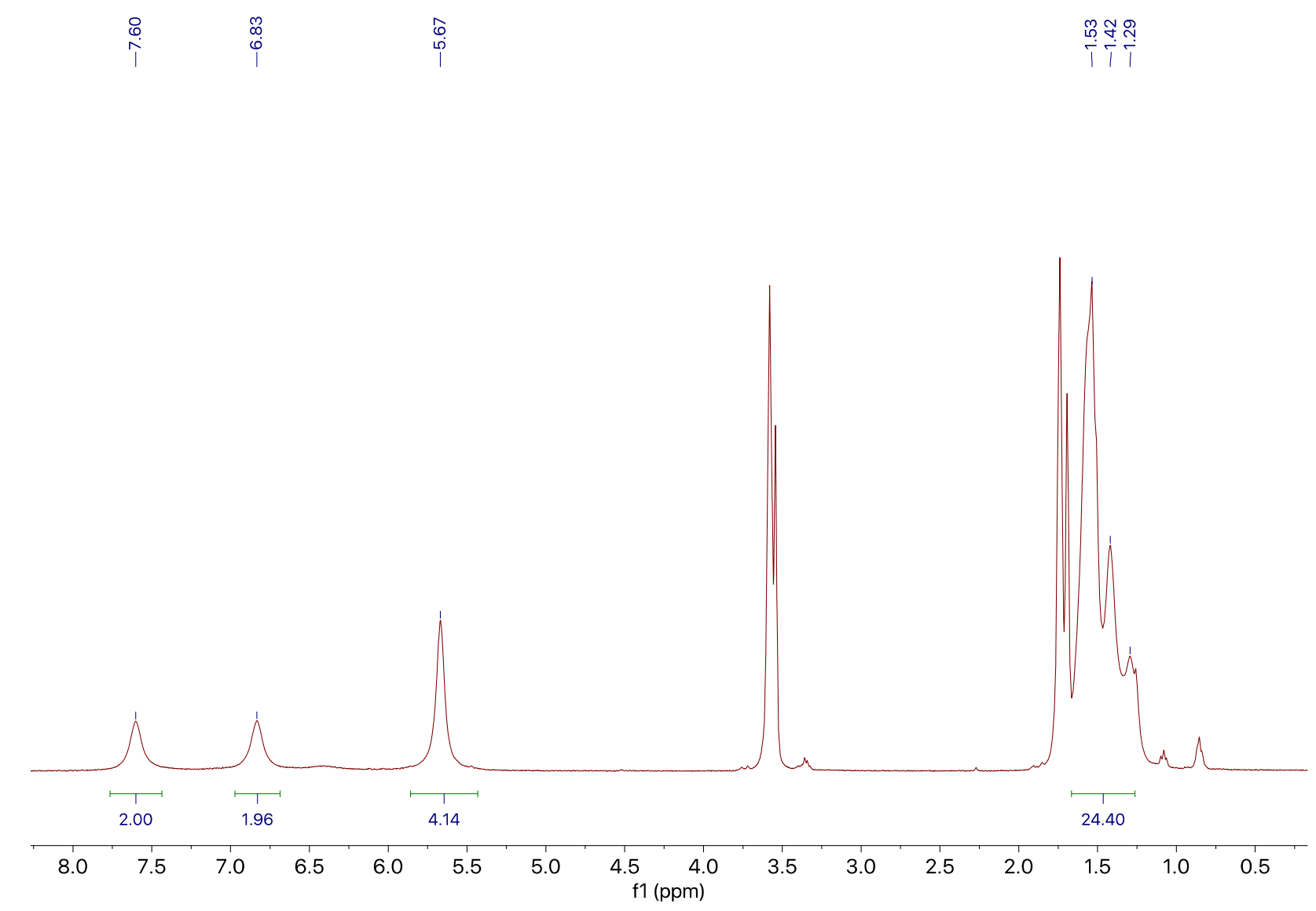

Figure S1. ${ }^{1} \mathrm{H}$ NMR spectrum of $[\mathrm{Li}(\mathrm{THF})]_{2}\left[\mathrm{~L}^{\Delta}\right](\mathbf{1})$ in THF- $d_{8}$ at room temperature. 


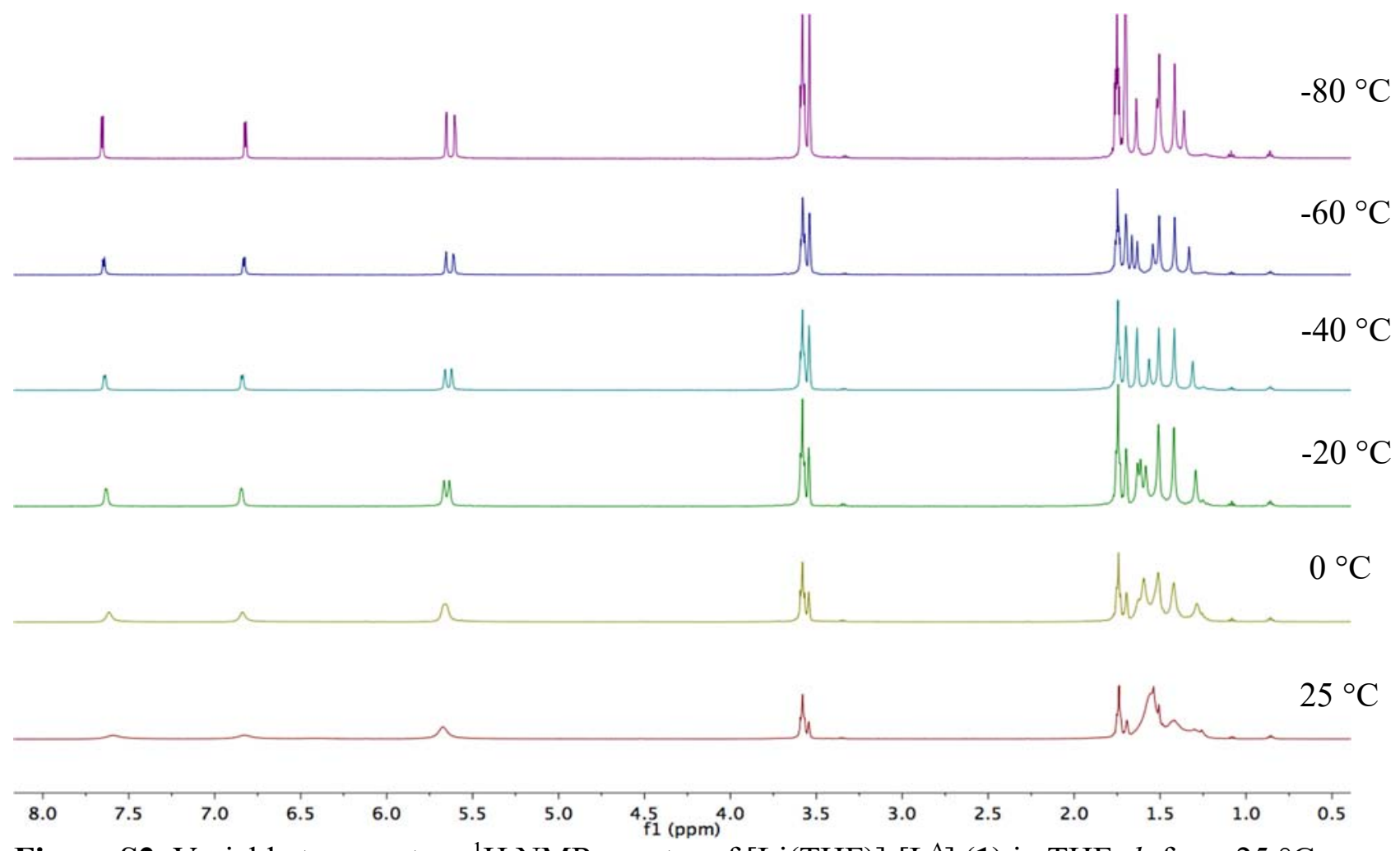

Figure S2. Variable temperature ${ }^{1} \mathrm{H}$ NMR spectra of $[\mathrm{Li}(\mathrm{THF})]_{2}\left[\mathrm{~L}^{\Delta}\right](\mathbf{1})$ in THF- $d 8$ from $25{ }^{\circ} \mathrm{C}$ (bottom) to $-80^{\circ} \mathrm{C}$ (top). 

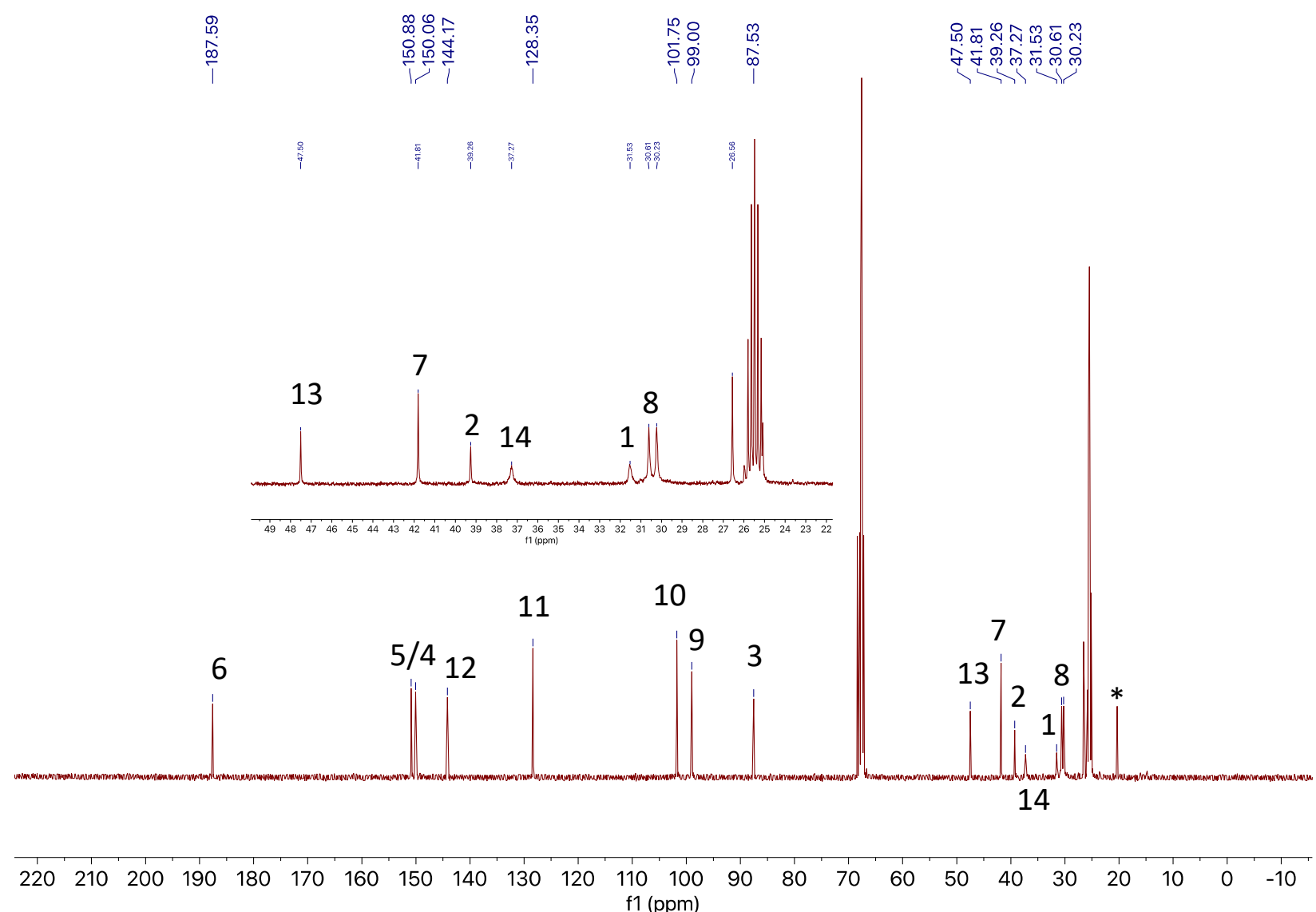
Figure S3. ${ }^{13} \mathrm{C}\left\{{ }^{1} \mathrm{H}\right\}$ NMR spectrum of $[\mathrm{Li}(\mathrm{THF})]_{2}\left[\mathrm{~L}^{\Delta}\right](\mathbf{1})$ in THF- $d 8$ at $-30{ }^{\circ} \mathrm{C}$. $(*)$ indicates and unidentified impurity.

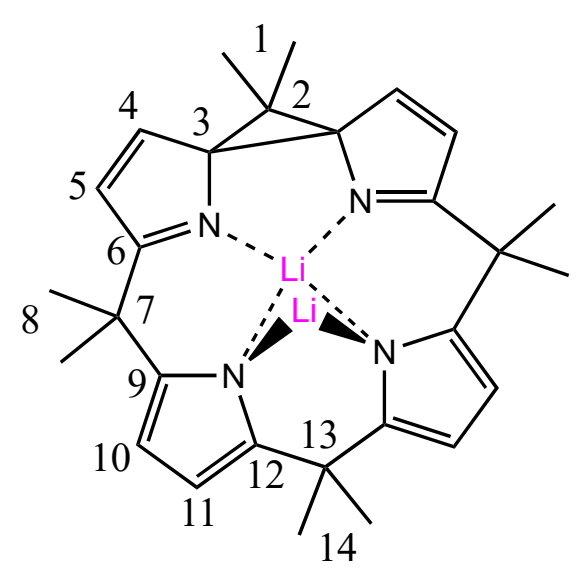



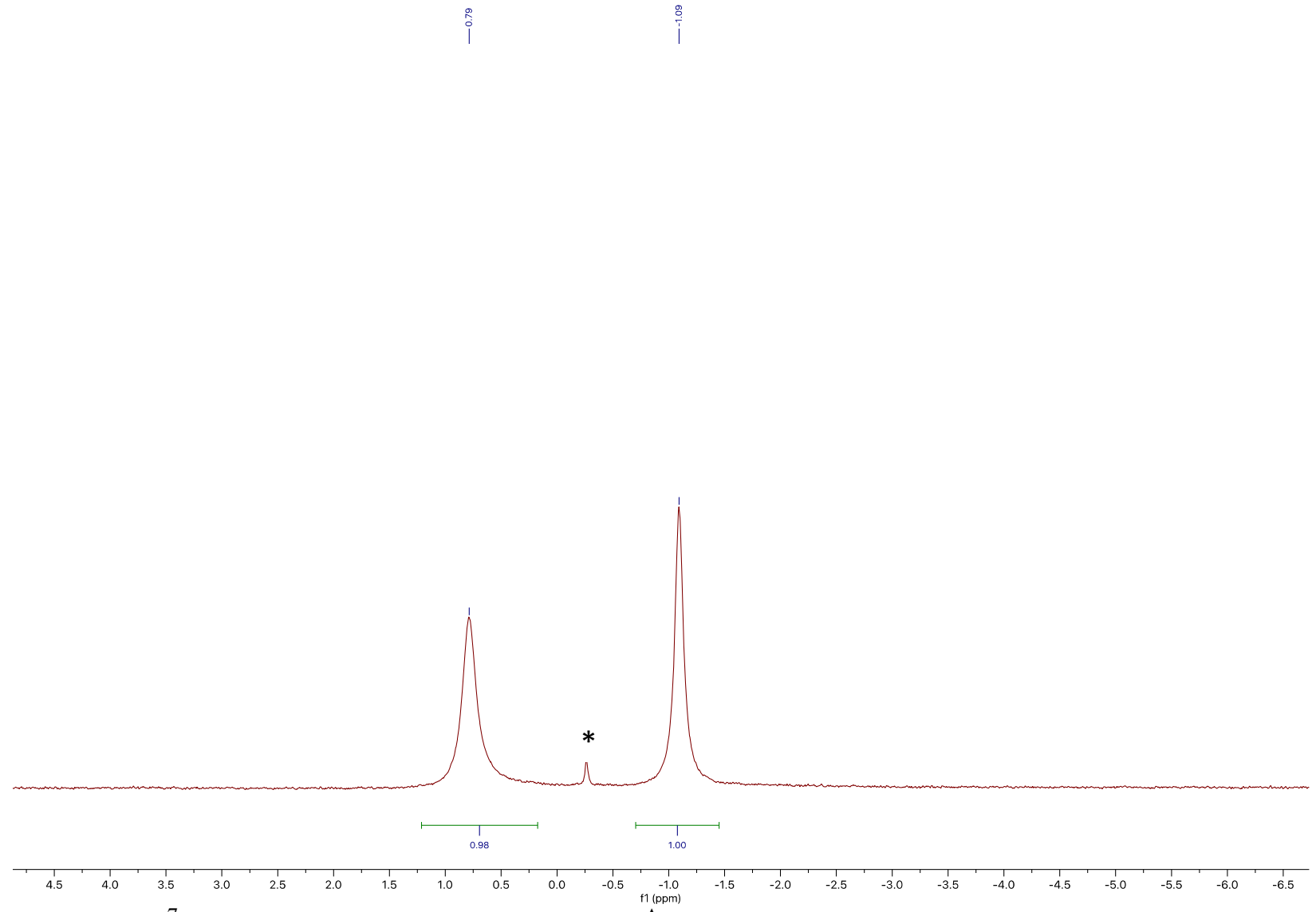

Figure S4. ${ }^{7} \mathrm{Li}$ NMR spectrum of $[\mathrm{Li}(\mathrm{THF})]_{2}\left[\mathrm{~L}^{\Delta}\right](\mathbf{1})$ in THF- $d_{8}$ at room temperature. $(*)$

indicates the resonance assignable to LiI. 


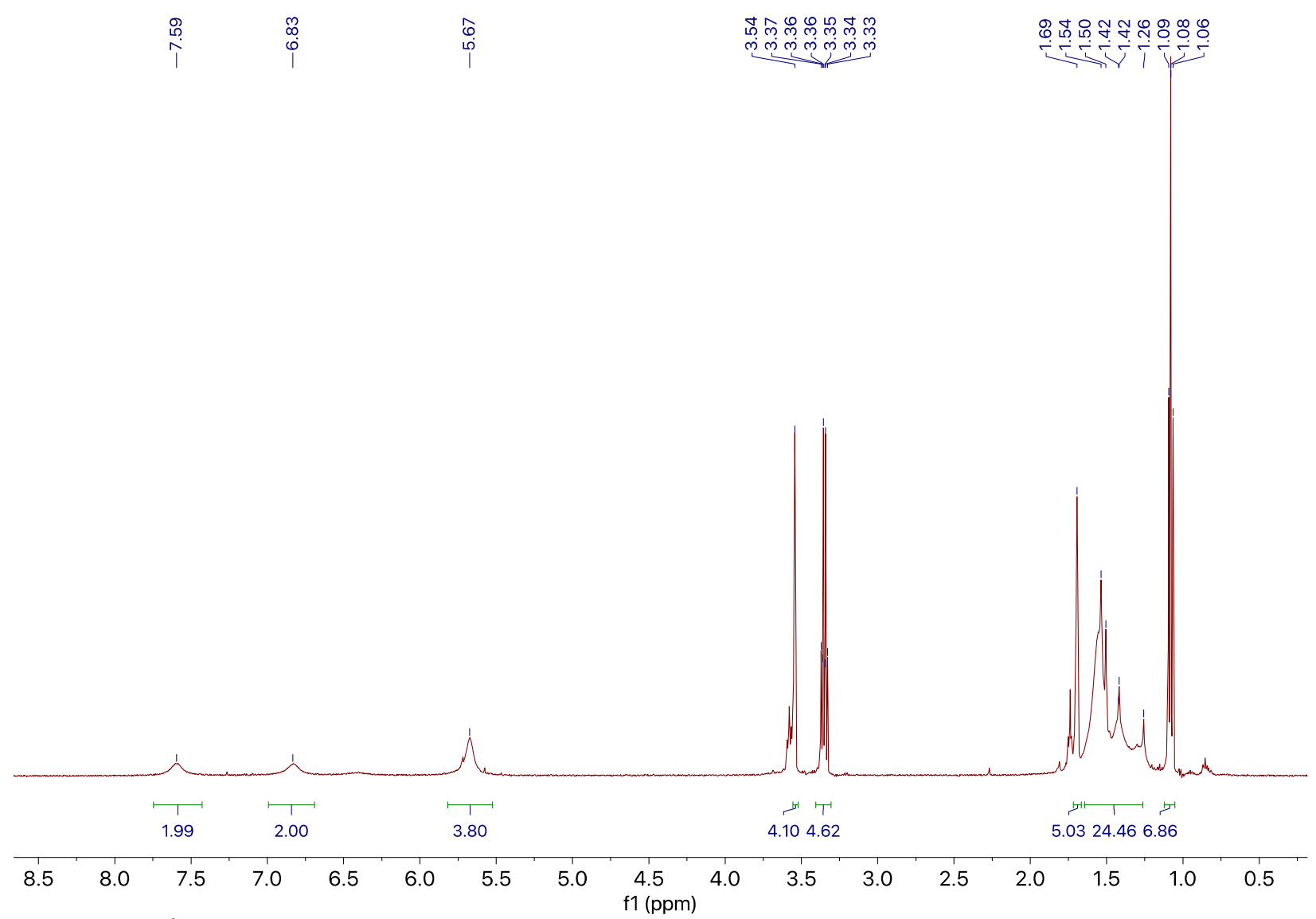

Figure S5. ${ }^{1} \mathrm{H}$ NMR spectrum of isolated crystalline material of

$[\mathrm{Li}(\mathrm{THF})]\left[\mathrm{Li}(\mathrm{THF})_{0.58}\left(\mathrm{Et}_{2} \mathrm{O}\right)_{0.42}\right]\left[\mathrm{L}^{\Delta}\right]$ in $\mathrm{THF}-d_{8}$ at room temperature. 


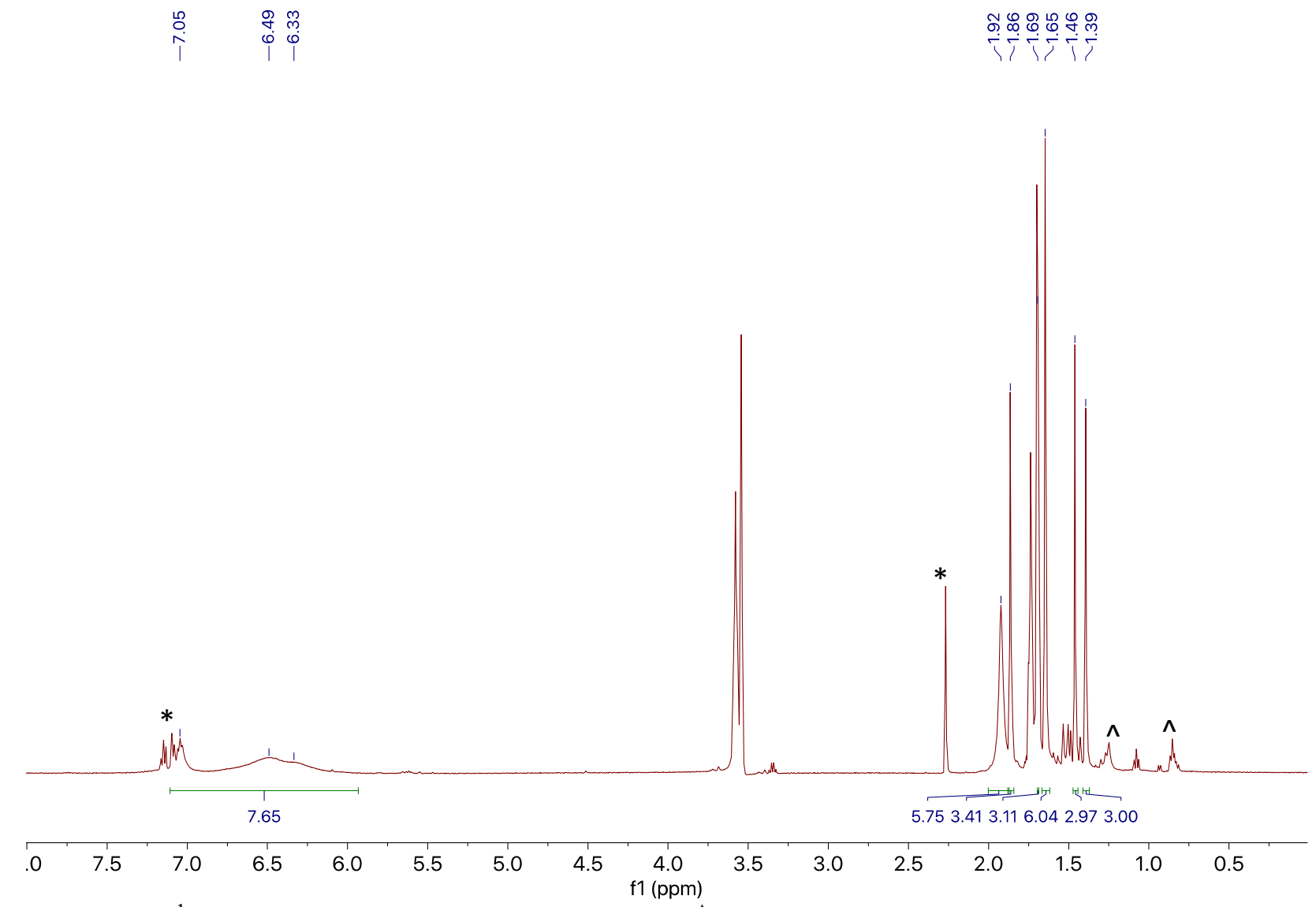

Figure S6. ${ }^{1} \mathrm{H}$ NMR spectrum of $[\mathrm{Li}(\mathrm{THF})]\left[\mathrm{UO}_{2}\left(\mathrm{~L}^{\Delta}\right) \mathrm{Cl}(\mathrm{THF})\right](2)$ in THF- $d_{8}$ at room temperature.

$(*)$ indicates resonances assignable to toluene, $\left({ }^{\wedge}\right)$ indicates resonances assignable to hexanes. 


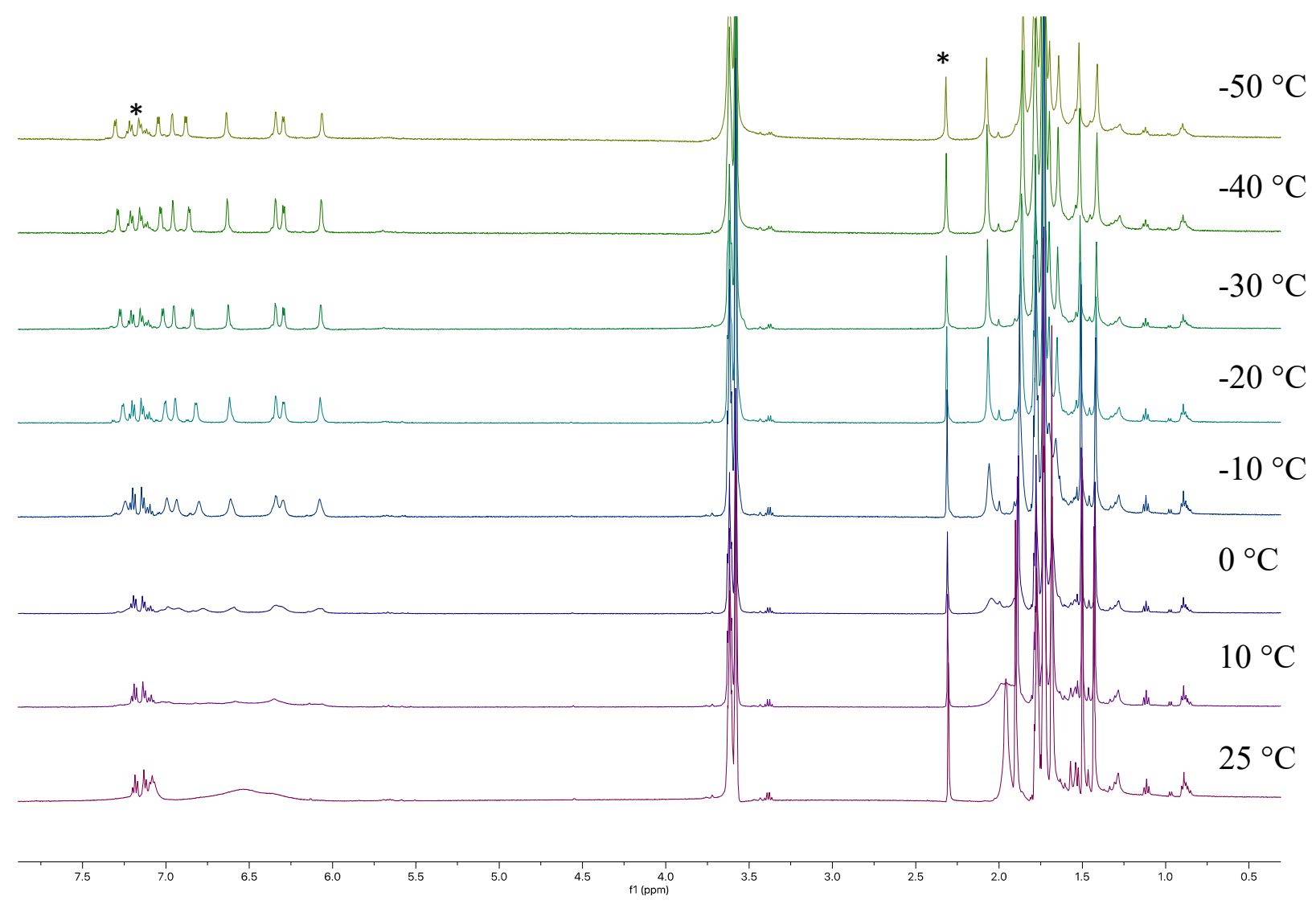

Figure S7. Variable temperature ${ }^{1} \mathrm{H}$ NMR spectra of $[\mathrm{Li}(\mathrm{THF})]\left[\mathrm{UO}_{2}\left(\mathrm{~L}^{\Delta}\right) \mathrm{Cl}(\mathrm{THF})\right](2)$ in $\mathrm{THF}-d_{8}$ from $25^{\circ} \mathrm{C}$ (bottom) to $-50^{\circ} \mathrm{C}$ (top). (*) indicates the resonances assignable to toluene. 


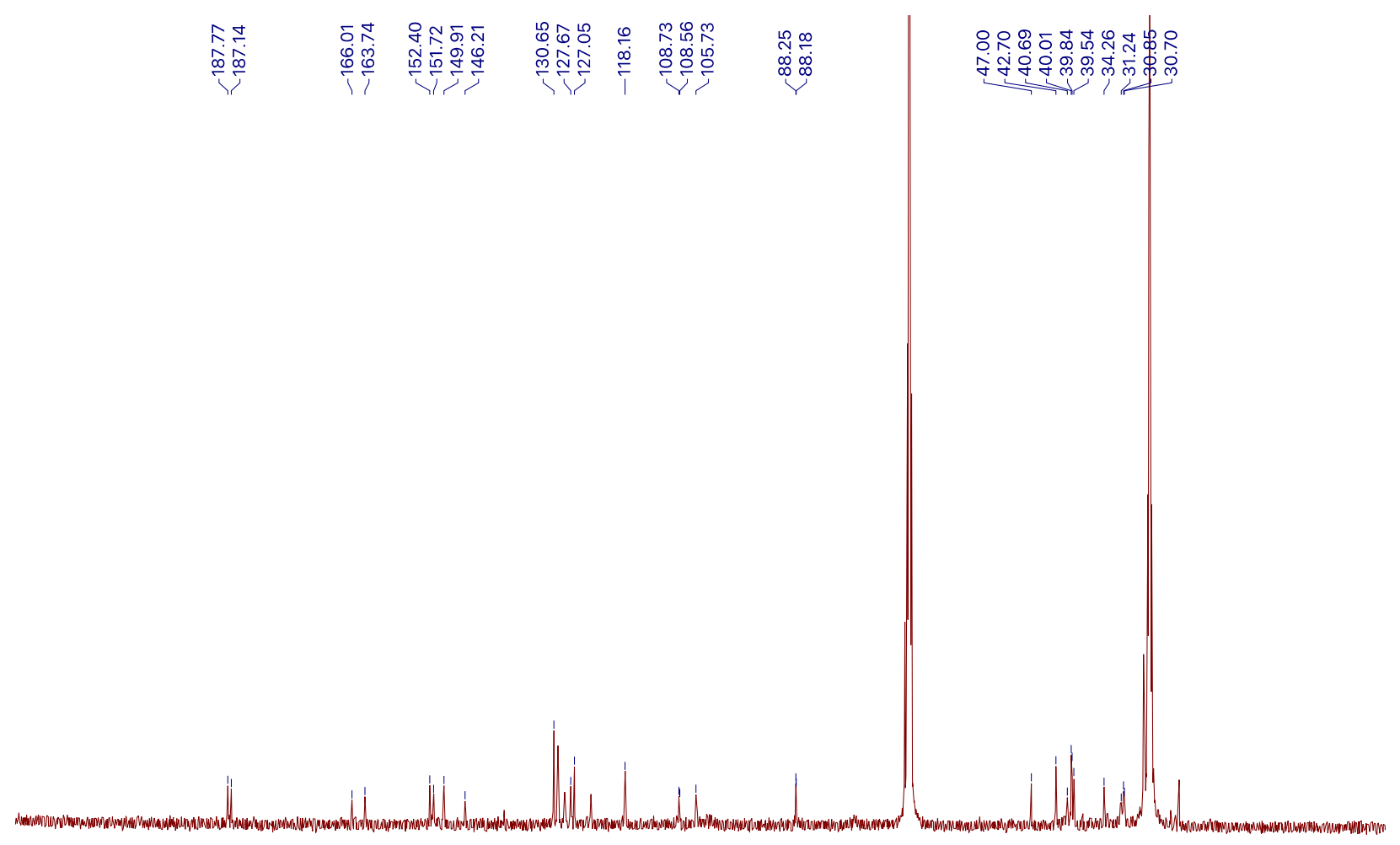

$\begin{array}{llllllllllllllllllllllll}220 & 210 & 200 & 190 & 180 & 170 & 160 & 150 & 140 & 130 & 120 & 110 & 100 & 90 & 80 & 70 & 60 & 50 & 40 & 30 & 20 & 10 & 0 & -10\end{array}$ Figure S8. ${ }^{13} \mathrm{C}\left\{{ }^{1} \mathrm{H}\right\}$ NMR spectrum of $[\mathrm{Li}(\mathrm{THF})]\left[\mathrm{UO}_{2}\left(\mathrm{~L}^{\Delta}\right) \mathrm{Cl}(\mathrm{THF})\right](2)$ in THF- $d 8$ at $-30{ }^{\circ} \mathrm{C}$. 


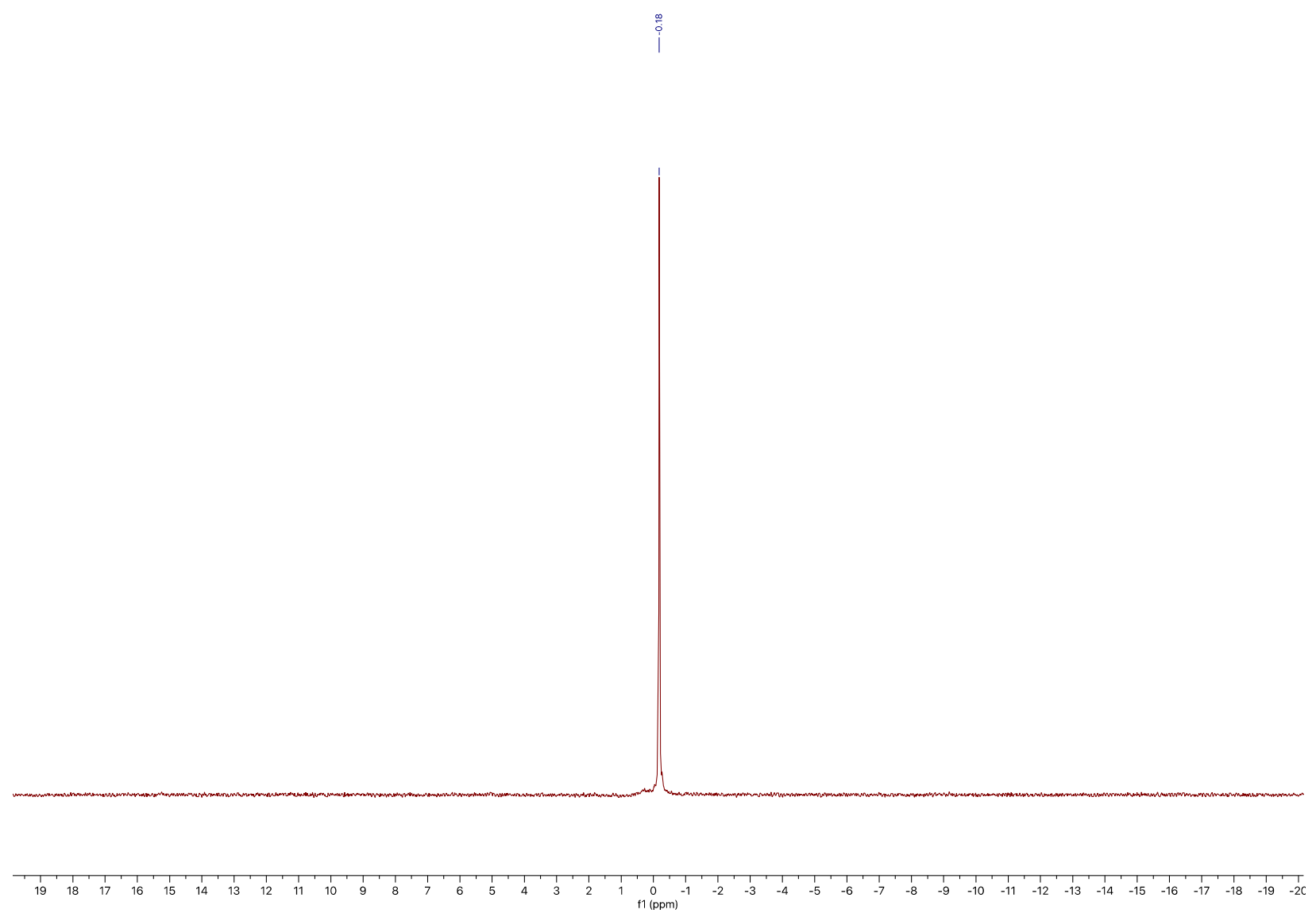

Figure S9. ${ }^{7} \mathrm{Li} \mathrm{NMR}$ spectrum of $[\mathrm{Li}(\mathrm{THF})]\left[\mathrm{UO}_{2}\left(\mathrm{~L}^{\Delta}\right) \mathrm{Cl}(\mathrm{THF})\right](\mathbf{2})$ in $\mathrm{THF}-d_{8}$ at room temperature. 


$$
\begin{array}{lllll}
\hline & 9 & 0 & \stackrel{1}{N} & 0 \\
0 & 0 & 0 & 0 & 0 \\
1 & 1 & 1 & 1 & 1
\end{array}
$$

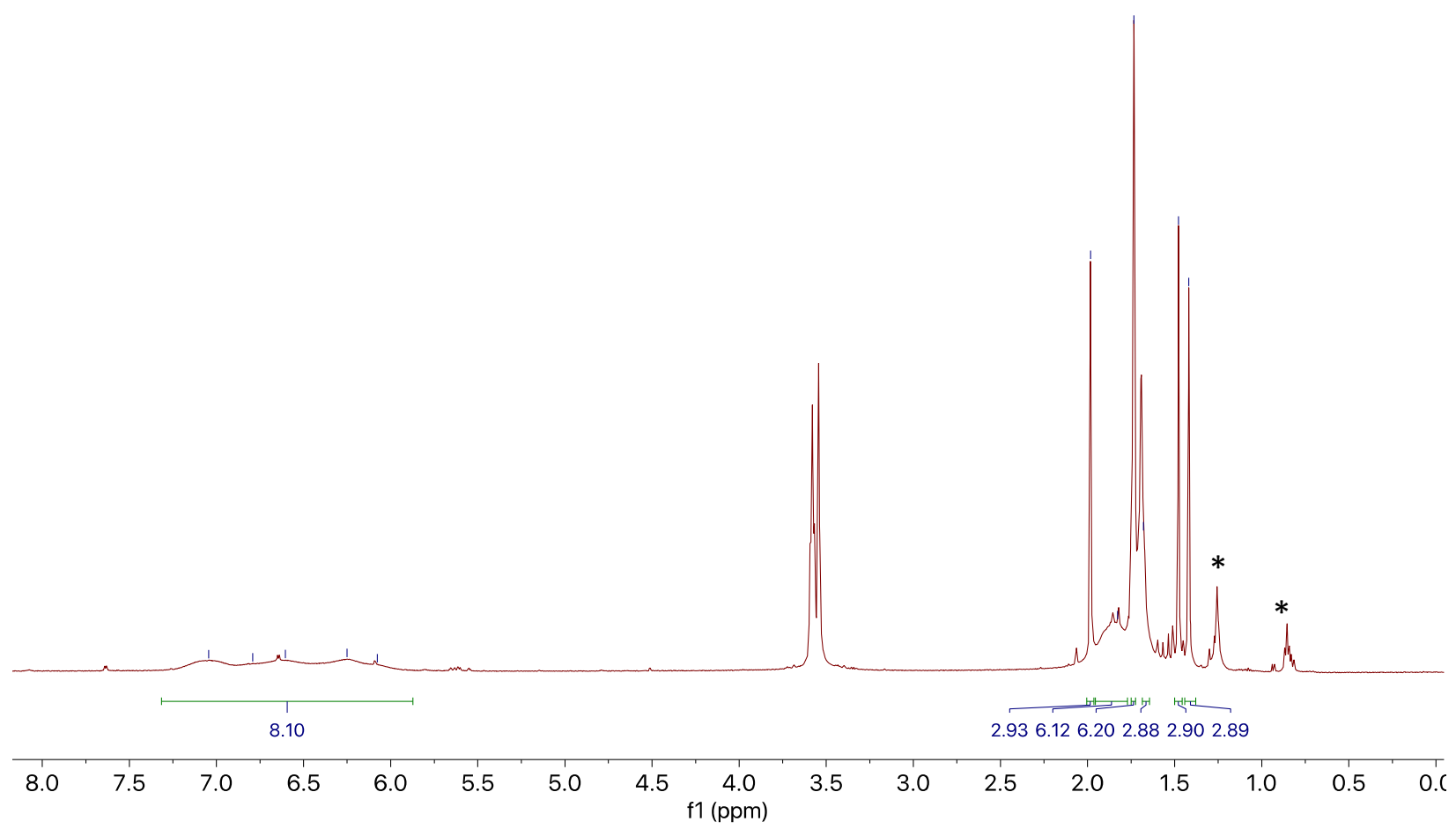

Figure S10. ${ }^{1} \mathrm{H}$ NMR spectrum of $[\mathrm{Li}(\mathrm{THF})]\left[\mathrm{UO}_{2}\left(\mathrm{~L}^{\Delta}\right)(\mathrm{OTf})(\mathrm{THF})\right](3)$ in $\mathrm{THF}-d_{8}$ at room temperature. $(*)$ indicates resonances assignable to hexanes. 


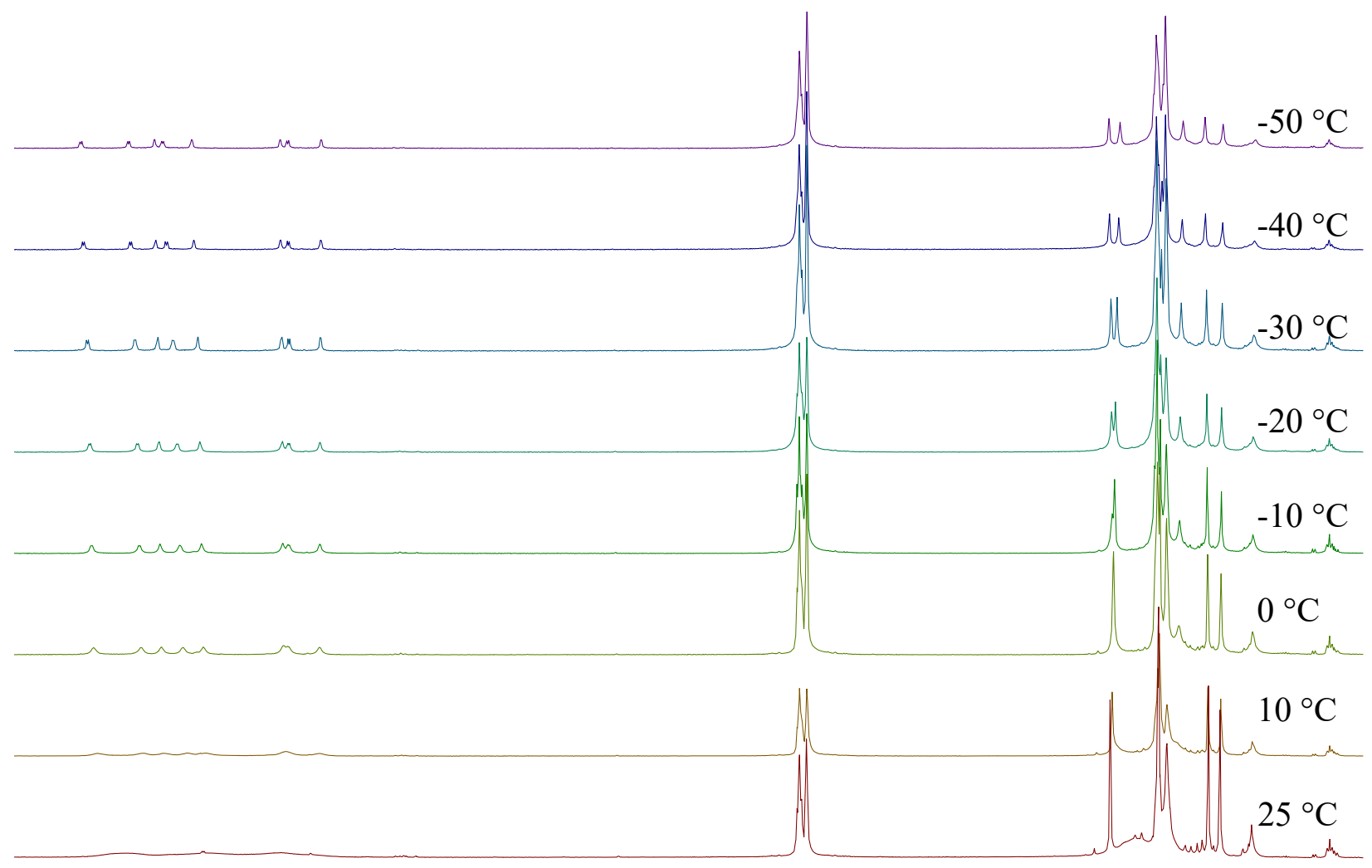

\begin{tabular}{llllllllllllllllllllllllllllllllllllllllllllllll}
\hline & 7.6 & 7.4 & 7.2 & 7.0 & 6.8 & 6.6 & 6.4 & 6.2 & 6.0 & 5.8 & 5.6 & 5.4 & 5.2 & 5.0 & 4.8 & 4.6 & 4.4 & 4.2 & 4.0 & 3.8 & 3.6 & 3.4 & 3.2 & 3.0 & 2.8 & 2.6 & 2.4 & 2.2 & 2.0 & 1.8 & 1.6 & 1.4 & 1.2 & 1.0 & 0.8 \\
\hline
\end{tabular} Figure S11. Variable temperature ${ }^{1} \mathrm{H}$ NMR spectra of $[\mathrm{Li}(\mathrm{THF})]\left[\mathrm{UO}_{2}\left(\mathrm{~L}^{\Delta}\right)(\mathrm{OTf})(\mathrm{THF})\right](3)$ in THF- $d_{8}$ from $25{ }^{\circ} \mathrm{C}$ (bottom) to $-50^{\circ} \mathrm{C}$ (top). 

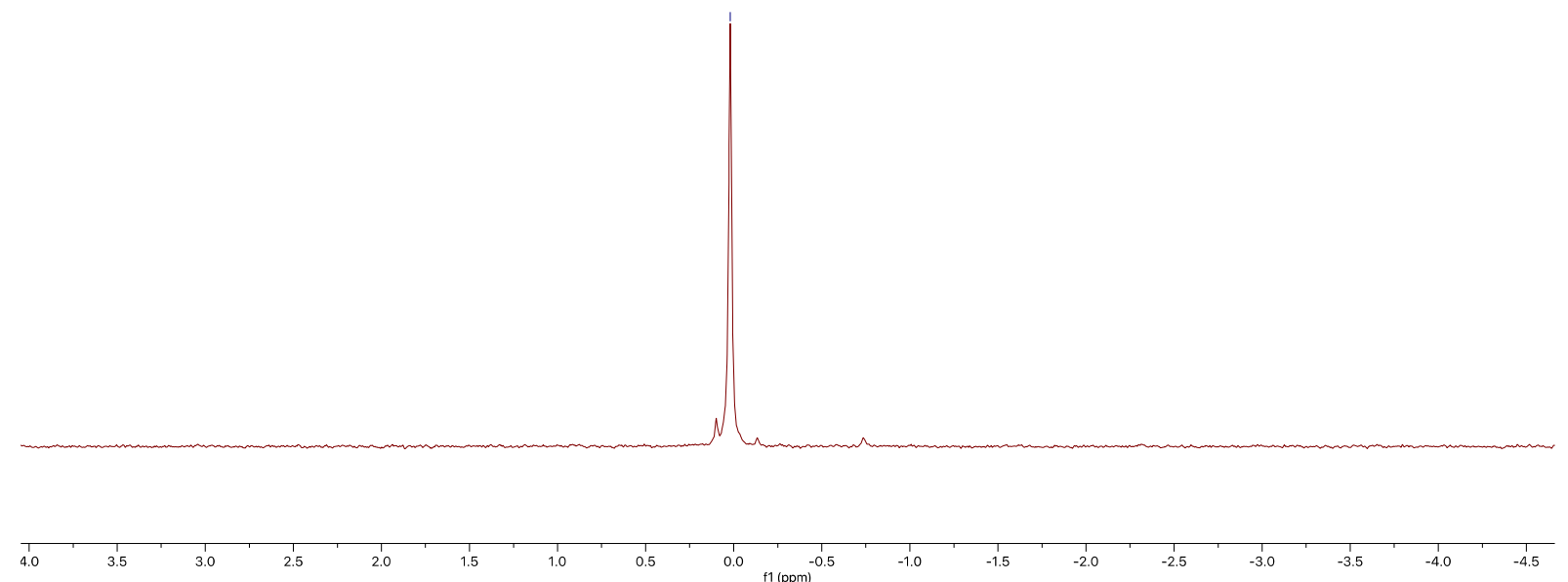

Figure S12. ${ }^{7} \mathrm{Li}$ NMR spectrum of $[\mathrm{Li}(\mathrm{THF})]\left[\mathrm{UO}_{2}\left(\mathrm{~L}^{\Delta}\right)(\mathrm{OTf})(\mathrm{THF})\right](3)$ in THF- $d 8$ at room temperature. 


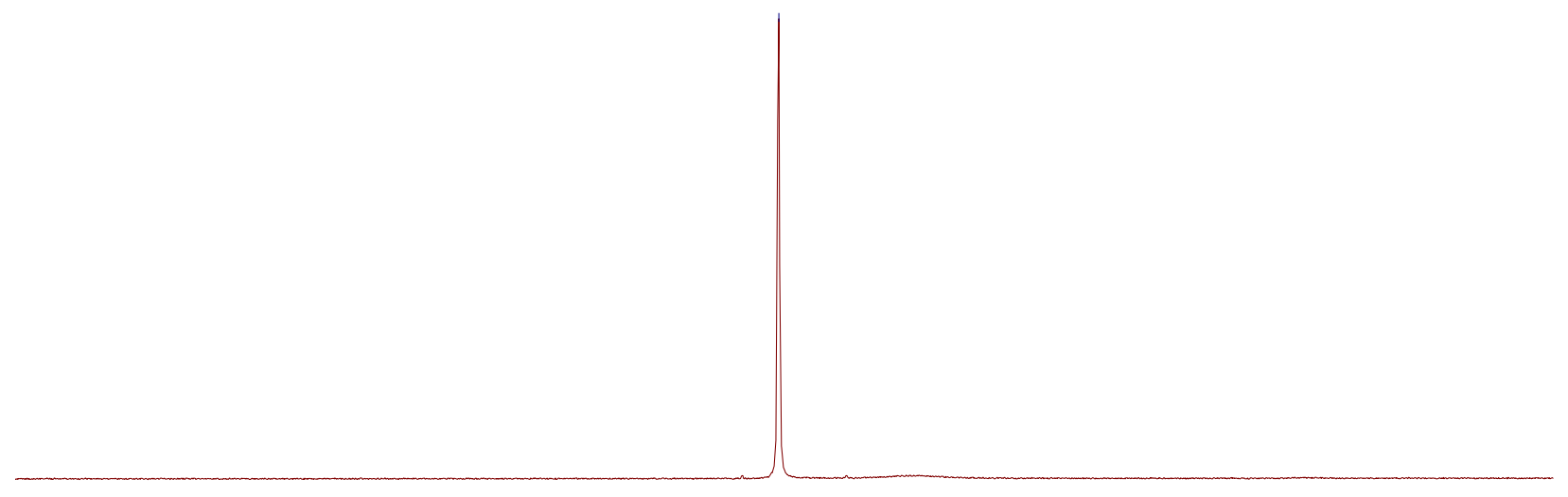

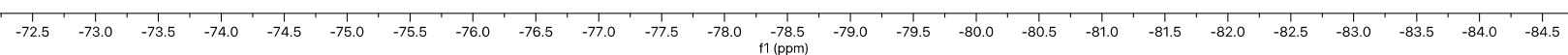
Figure S13. ${ }^{19} \mathrm{~F}\left\{{ }^{1} \mathrm{H}\right\}$ NMR spectrum of $[\mathrm{Li}(\mathrm{THF})]\left[\mathrm{UO}_{2}\left(\mathrm{~L}^{\Delta}\right)(\mathrm{OTf})(\mathrm{THF})\right](3)$ in THF- $d_{8}$ at room temperature. 


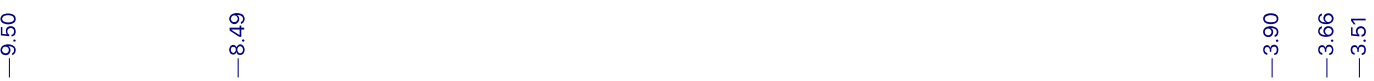

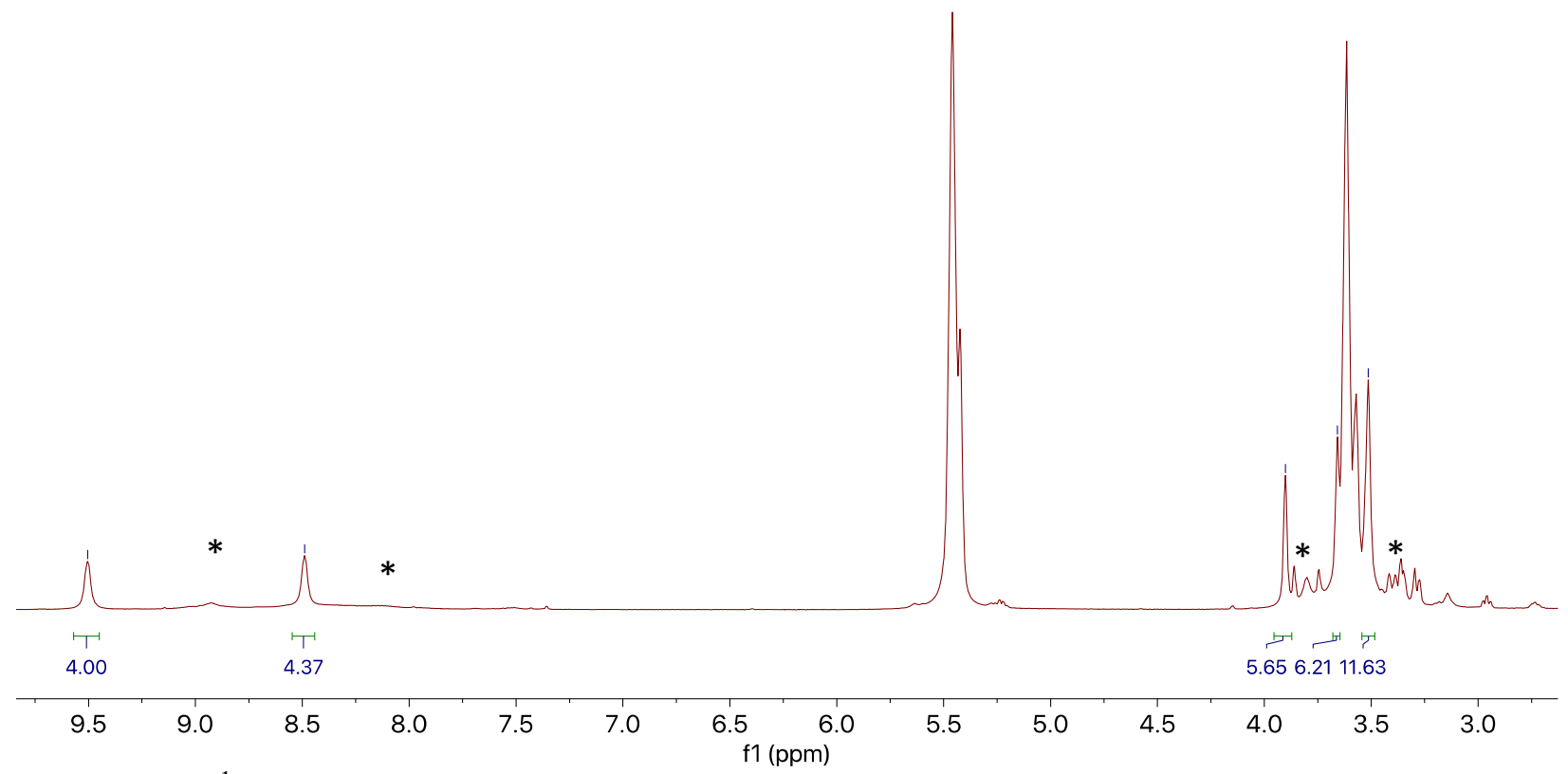

Figure S14. ${ }^{1} \mathrm{H}$ NMR spectrum of the brown solid isolated during the work up of

$[\mathrm{Li}(\mathrm{THF})]\left[\mathrm{UO}_{2}\left(\mathrm{~L}^{\Delta}\right)(\mathrm{OTf})(\mathrm{THF})\right](\mathbf{3})$ in THF- $d 8$ at room temperature. $\left(^{*}\right)$ indicates resonances assignable to 3 . Resonances with integrations are assigned to $\mathrm{L}^{\Delta \Delta} \cdot 13,14$ 


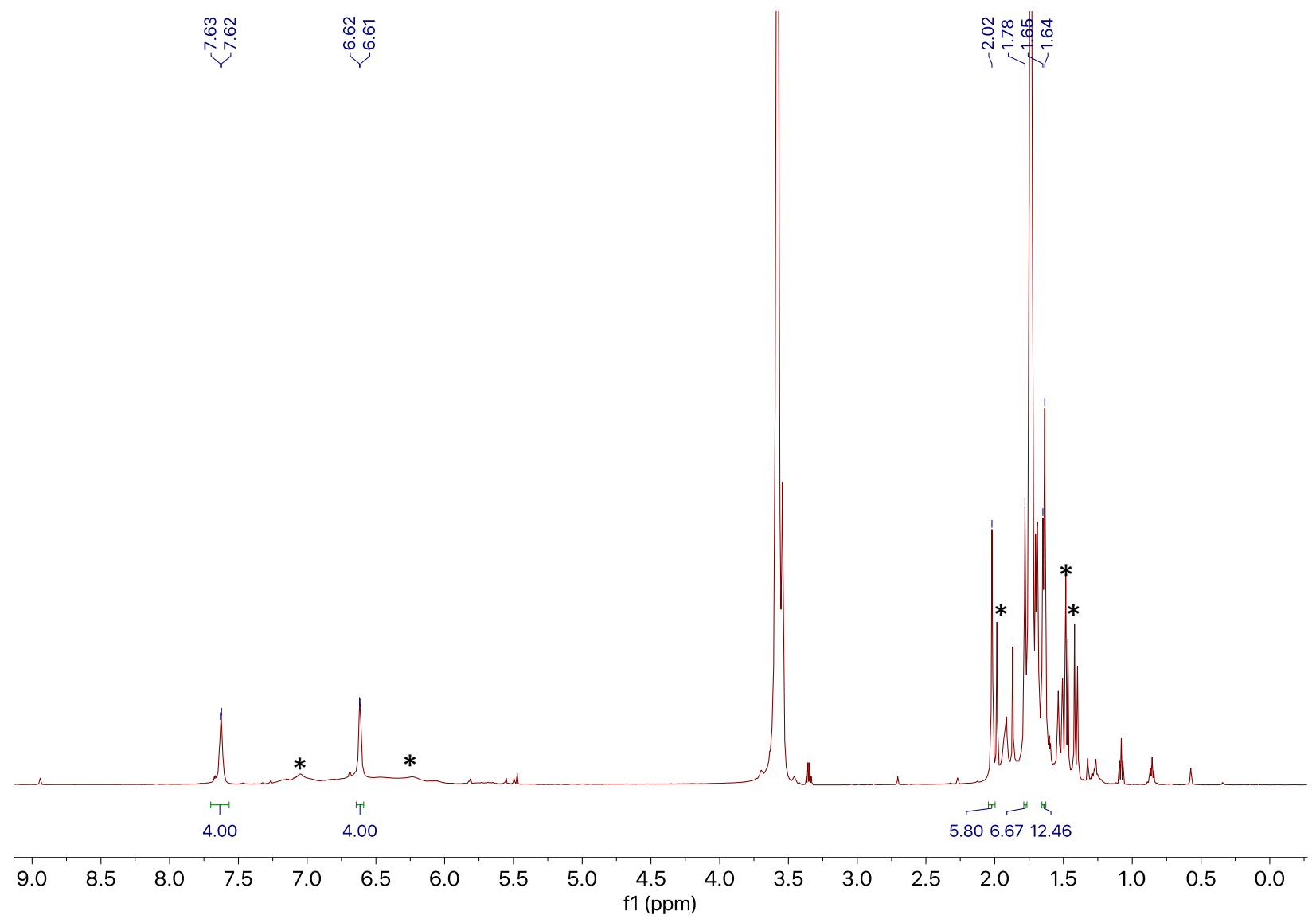

Figure S15. ${ }^{1} \mathrm{H}$ NMR spectrum of a crude reaction mixture of $\left[\mathrm{UO}_{2}(\mathrm{OTf})_{2}(\mathrm{THF})_{3}\right]$ with 1 in THF- $d 8$ at room temperature. Resonances with integrations are assigned to $\mathrm{L}^{\Delta \Delta} \cdot{ }^{13,14}$ Also present in the spectrum is $[\mathrm{Li}(\mathrm{THF})]\left[\mathrm{UO}_{2}\left(\mathrm{~L}^{\Delta}\right)(\mathrm{OTf})(\mathrm{THF})\right](3)$, indicated by $(*)$. 

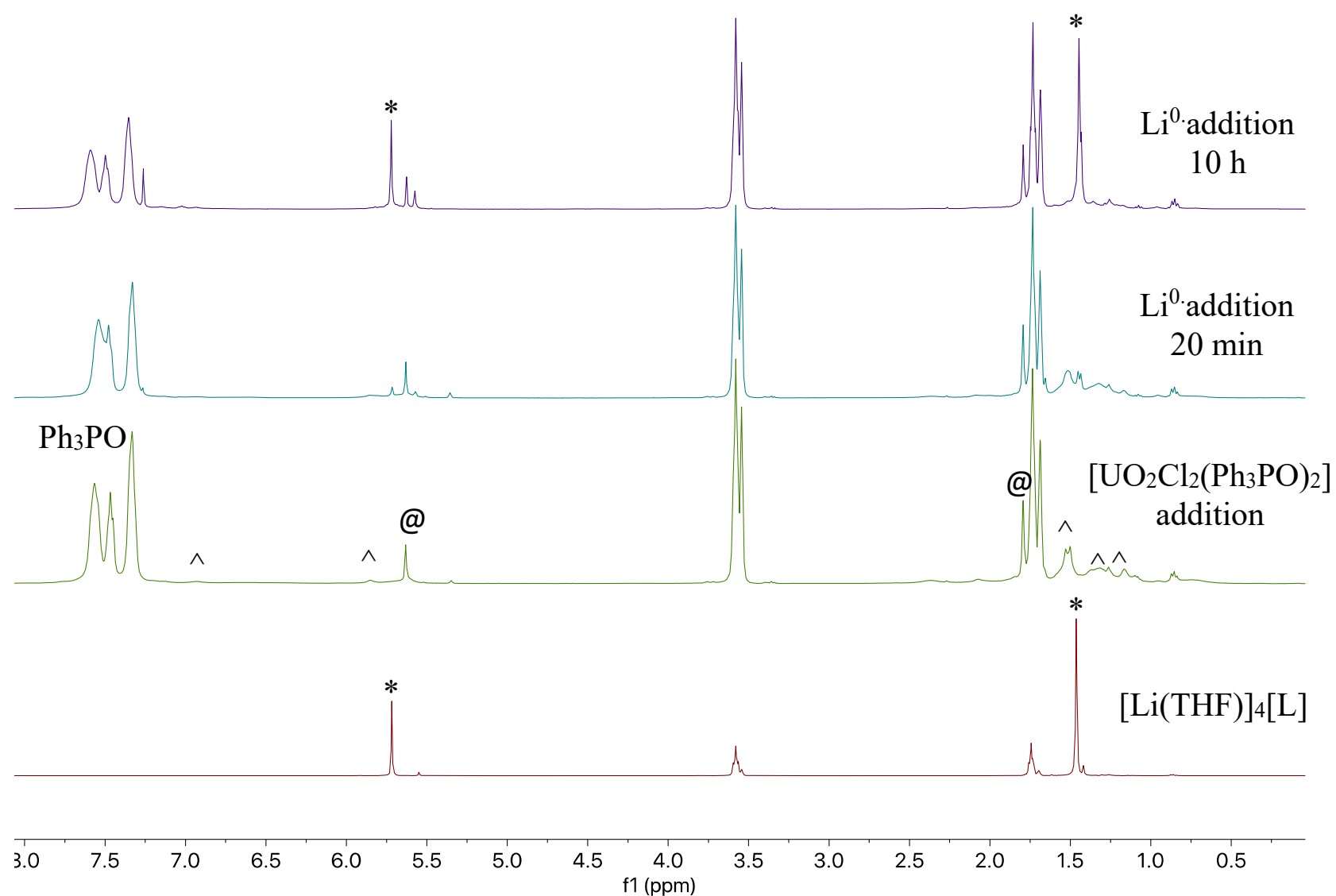

Figure S16. ${ }^{1} \mathrm{H}$ NMR spectra of a reaction of $[\mathrm{Li}(\mathrm{THF})]_{4}[\mathrm{~L}]$ with $\left[\mathrm{UO}_{2} \mathrm{Cl}_{2}\left(\mathrm{Ph}_{3} \mathrm{PO}\right)_{2}\right]$ followed by reaction with $\mathrm{Li}^{0}$ metal in $\mathrm{THF}-d_{8}$ at room temperature. $\left.{ }^{*}\right)$ indicates resonances assignable to $[\mathrm{Li}(\mathrm{THF})]_{4}[\mathrm{~L}],\left({ }^{\wedge}\right)$ indicates resonances assignable to 1, $(@)$ indicates resonances assignable to $\mathrm{H}_{4} \mathrm{~L}$. 
Table S1. Crystallographic details for complexes $[\mathrm{Li}(\mathrm{THF})]\left[\mathrm{Li}(\mathrm{THF})_{0.58}\left(\mathrm{Et}_{2} \mathrm{O}\right)_{0.42}\right]\left[\mathrm{L}^{\Delta}\right] \cdot 0.5 \mathrm{Et}_{2} \mathrm{O}$,

$2 \cdot \mathrm{C}_{7} \mathrm{H}_{8}, 3$.

\begin{tabular}{|c|c|c|c|}
\hline & $\begin{array}{l}{[\mathrm{Li}(\mathrm{THF})]\left[\mathrm{Li}(\mathrm{THF})_{0.58}\left(\mathrm{Et}_{2} \mathrm{O}\right.\right.} \\
\left.{ }_{0.42}\right]\left[\mathrm{L}^{\Delta}\right] \cdot 0.5 \mathrm{Et}_{2} \mathrm{O}\end{array}$ & $2 \cdot \mathrm{C}_{7} \mathrm{H}_{8}$ & 3 \\
\hline Formula & $\mathrm{C}_{38} \mathrm{H}_{48.84} \mathrm{~N}_{4} \mathrm{O}_{2.5} \mathrm{Li}_{2}$ & $\mathrm{C}_{79} \mathrm{H}_{104} \mathrm{~N}_{8} \mathrm{O}_{8} \mathrm{Cl}_{2} \mathrm{Li}_{2} \mathrm{U}_{2}$ & $\mathrm{C}_{37} \mathrm{H}_{48} \mathrm{~N}_{4} \mathrm{O}_{7} \mathrm{SF}_{3} \mathrm{LiU}$ \\
\hline Crystal Habit, Color & Block, Orange & Needle, Dark-Green & Needle, Dark-Green \\
\hline Crystal Size (mm) & $0.2 \times 0.15 \times 0.05$ & $0.2 \times 0.05 \times 0.03$ & $0.25 \times 0.05 \times 0.03$ \\
\hline MW (g/mol) & 615.52 & 1854.54 & 994.82 \\
\hline crystal system & Monoclinic & Triclinic & Monoclinic \\
\hline space group & $\mathrm{C} 2 / \mathrm{c}$ & P-1 & $\mathrm{P} 2{ }_{1} / \mathrm{n}$ \\
\hline $\mathrm{a}(\AA)$ & $23.649(4)$ & $14.900(1)$ & $10.174(1)$ \\
\hline b $(\AA)$ & $19.253(3)$ & $16.145(1)$ & 19.791(1) \\
\hline c $(\AA)$ & $19.371(6)$ & $19.041(1)$ & $18.883(2)$ \\
\hline$\alpha\left(^{\circ}\right)$ & 90 & $65.066(2)$ & 90 \\
\hline$\beta\left(^{\circ}\right)$ & $124.753(8)$ & $89.891(2)$ & $94.163(5)$ \\
\hline$\gamma\left({ }^{\circ}\right)$ & 90 & $70.221(2)$ & 90 \\
\hline$V\left(\AA^{3}\right)$ & $7247(3)$ & $3855.0(4)$ & $3792.1(5)$ \\
\hline Z & 8 & 2 & 4 \\
\hline $\mathrm{T}(\mathrm{K})$ & $100(2)$ & $100(2)$ & $100(2)$ \\
\hline$\lambda(\AA)$ & 0.71073 & 0.71073 & 0.71073 \\
\hline GOF & 1.191 & 0.951 & 1.017 \\
\hline Density (calcd) $\left(\mathrm{Mg} / \mathrm{m}^{3}\right)$ & 1.128 & 1.598 & 1.743 \\
\hline $\begin{array}{l}\text { Absorption coefficient } \\
\left(\mathrm{mm}^{-1}\right)\end{array}$ & 0.070 & 4.324 & 4.404 \\
\hline $\mathrm{F}_{000}$ & 2647 & 1844 & 1968 \\
\hline Total no Reflections & 14163 & 13600 & 21756 \\
\hline Unique Reflections & 6094 & 10303 & 6442 \\
\hline Final R indices* & $\begin{array}{l}\mathrm{R}_{1}=0.0776 \\
\mathrm{wR}_{2}=0.1962\end{array}$ & $\begin{array}{l}\mathrm{R}_{1}=0.0999 \\
\mathrm{wR}_{2}=0.2549\end{array}$ & $\begin{array}{l}\mathrm{R}_{1}=0.0372 \\
\mathrm{wR}_{2}=0.0744\end{array}$ \\
\hline $\begin{array}{l}\text { Largest Diff. peak and } \\
\text { hole }\left(\mathrm{e}^{-} \mathrm{A}^{-3}\right)\end{array}$ & $0.744,-0.437$ & $7.045,-5.775$ & $1.681,-1.039$ \\
\hline
\end{tabular}




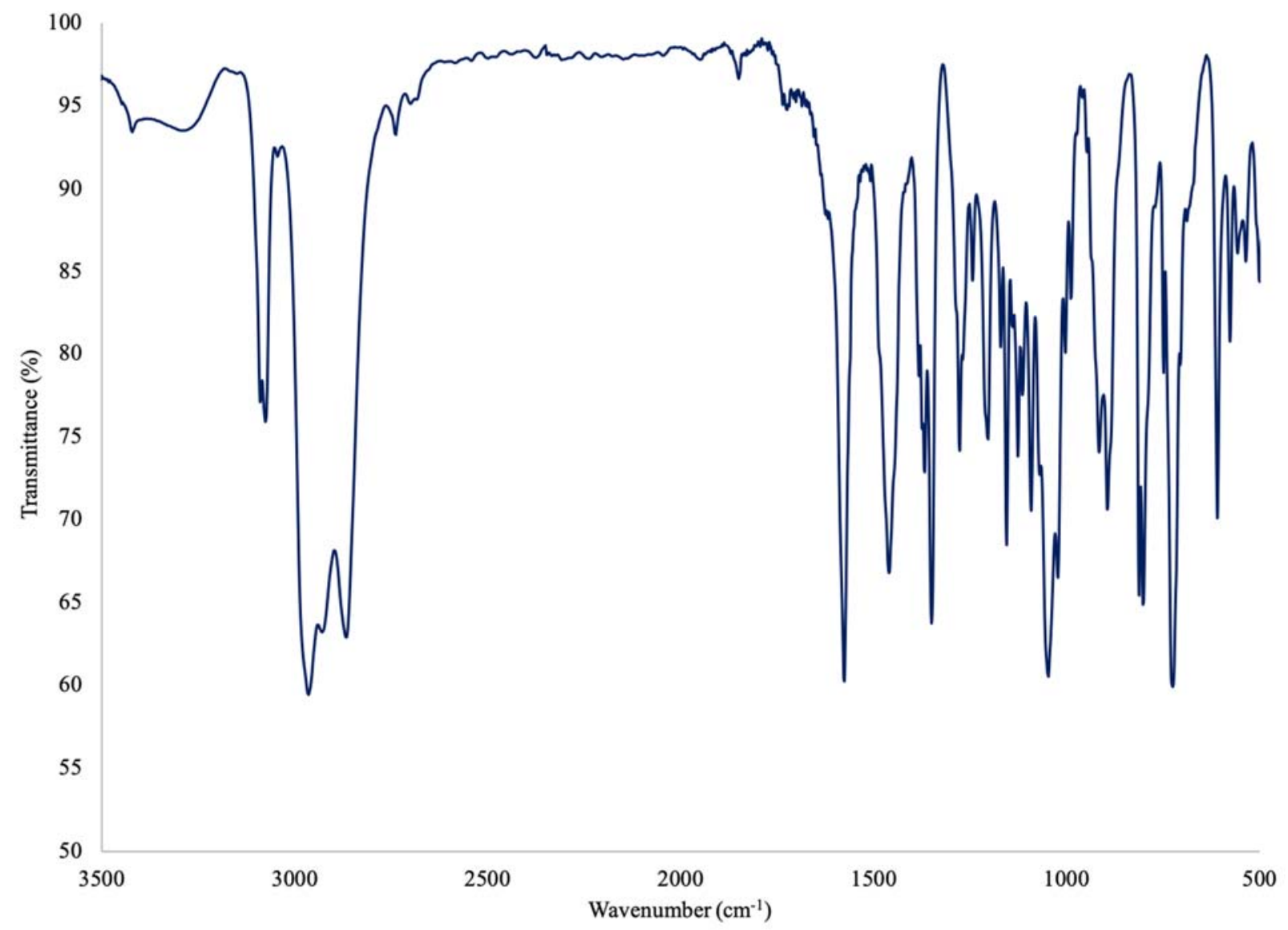

Figure S17. IR spectrum of $[\mathrm{Li}(\mathrm{THF})]_{2}\left[\mathrm{~L}^{\Delta}\right](\mathbf{1})(\mathrm{KBr}$ Pellet). 


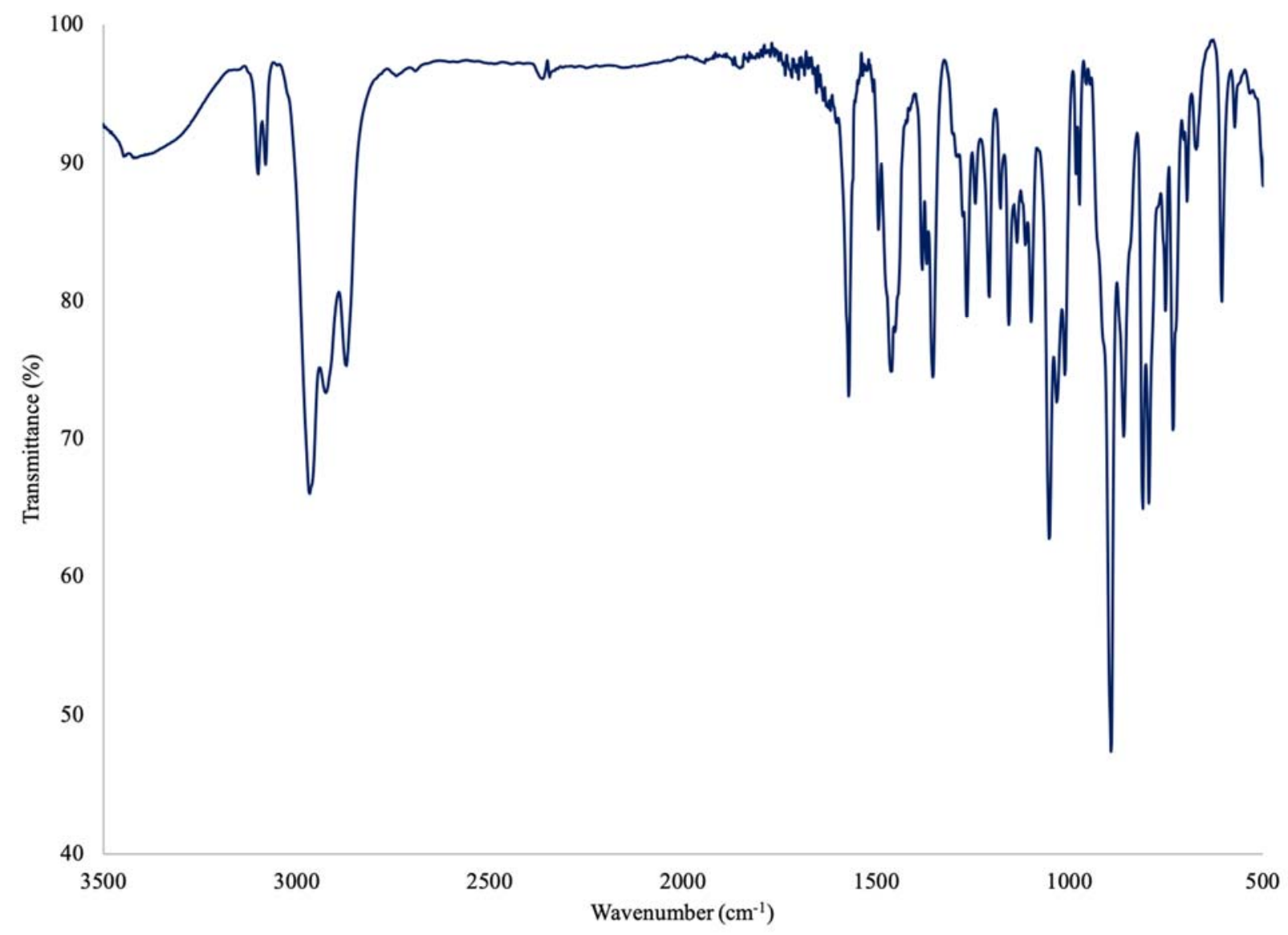

Figure S18. IR spectrum of $[\mathrm{Li}(\mathrm{THF})]\left[\mathrm{UO}_{2}\left(\mathrm{~L}^{\Delta}\right) \mathrm{Cl}(\mathrm{THF})\right]$ (2) (KBr Pellet). 


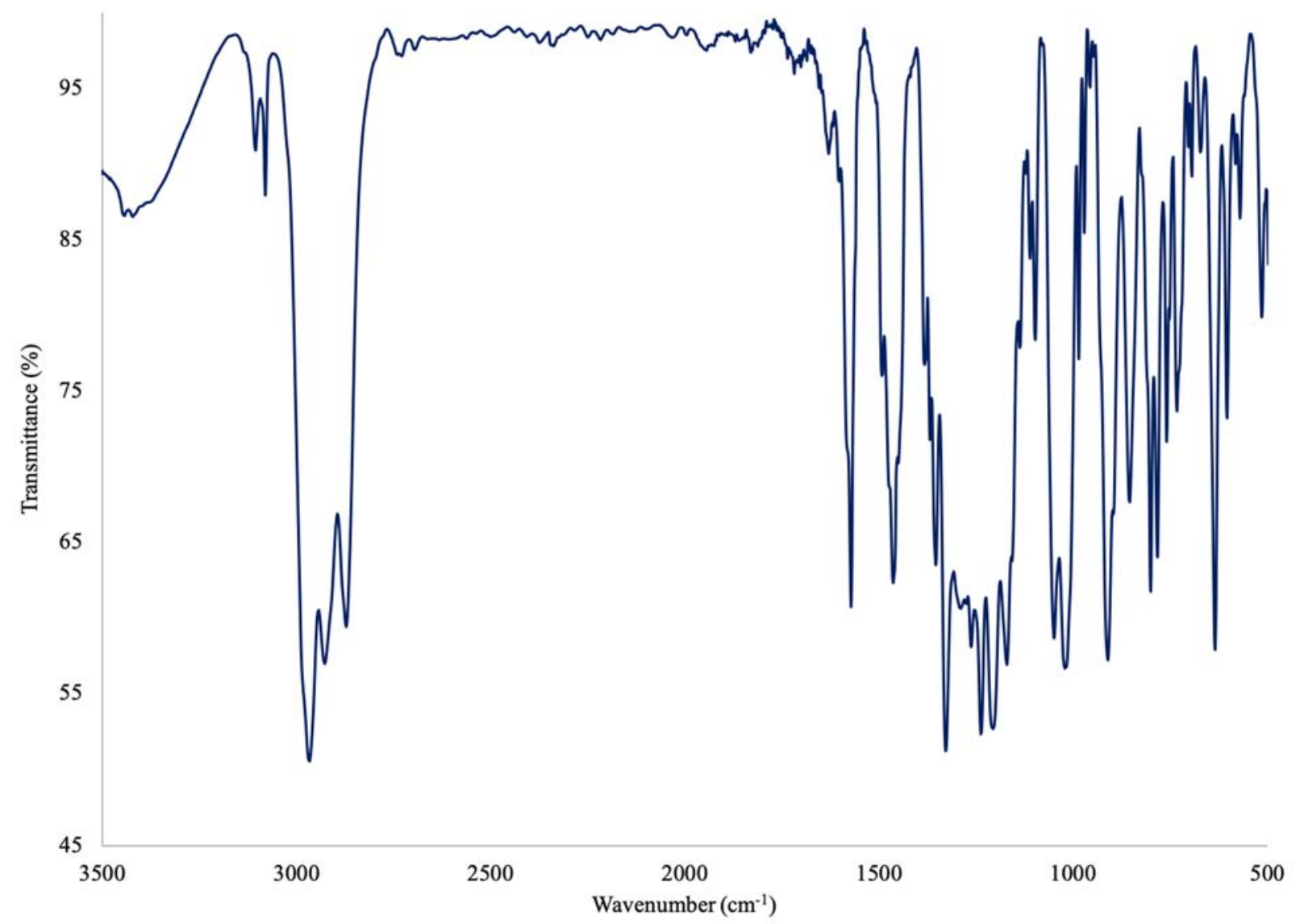

Figure S19. IR spectrum of $[\mathrm{Li}(\mathrm{THF})]\left[\mathrm{UO}_{2}\left(\mathrm{~L}^{\Delta}\right)(\mathrm{OTf})(\mathrm{THF})\right](3)$ (KBr Pellet). 


\section{References}

1. Wilkerson, M. P.; Burns, C. J.; Paine, R. T.; Scott, B. L., Synthesis and Crystal Structure of $\mathrm{UO}_{2} \mathrm{Cl}_{2}(\mathrm{THF})_{3}$ : A Simple Preparation of an Anhydrous Uranyl Reagent. Inorg. Chem. 1999, 38, 4156-4158.

2. Kiernicki, J. J.; Zeller, M.; Bart, S. C., Facile Reductive Silylation of $\mathrm{UO}_{2}{ }^{2+}$ to Uranium(IV) Chloride. Angew. Chem. Int. Ed. 2017, 56, 1097-1100.

3. Oldham, S. M.; Scott, B. L.; Oldham Jr, W. J., Reaction of the N-heterocyclic carbene, 1,3dimesityl- imidazol-2-ylidene, with a uranyl triflate complex, $\mathrm{UO}_{2}(\mathrm{OTf})_{2}(\text { thf })_{3}$. J. Appl. Organomet. Chem. 2006, 20, 39-43.

4. Jacoby, D.; Floriani, C.; Chiesi-Villa, A.; Rizzoli, C., Zirconium mesooctaethylporphyrinogen as a carrier for sodium hydride in toluene: zirconium-sodium bimetallic hydride and alkyls. J. Am. Chem. Soc. 1993, 115, 3595-3602.

5. De Angelis, S.; Solari, E.; Floriani, C.; Chiesi-Villa, A.; Rizzoli, C., Solvent-dependent forms of lithiated 5,5,10,10,15,15,20,20-octaethylporphyrinogen in solution and in the solid state and reaction with tetrahydrofuran. J. Chem. Soc., Dalton Trans. 1994, 2467-2469.

6. Harris, R. K.; Becker, E. D.; De Menezes, S. M. C.; Granger, P.; Hoffman, R. E.; Zilm, K. W., Further Conventions for NMR Shielding and Chemical Shifts (IUPAC Recommendations 2008). Magn. Reson. Chem. 2008, 46, 582-598.

7. Harris, R. K.; Becker, E. D.; Cabral de Menezes, S. M.; Goodfellow, R.; Granger, P., NMR nomenclature: nuclear spin properties and conventions for chemical shifts. IUPAC Recommendations 2001. International Union of Pure and Applied Chemistry. Physical Chemistry Division. Commission on Molecular Structure and Spectroscopy. Magn. Reson. Chem. 2002, 40, 489-505.

8. Seaman, L. A.; Hrobárik, P.; Schettini, M. F.; Fortier, S.; Kaupp, M.; Hayton, T. W., A Rare Uranyl(VI)-Alkyl Ate Complex $\left[\mathrm{Li}(\mathrm{DME})_{1.5}\right]_{2}\left[\mathrm{UO}_{2}\left(\mathrm{CH}_{2} \mathrm{SiMe}_{3}\right)_{4}\right]$ and Its Comparison with a Homoleptic Uranium(VI)-Hexaalkyl. Angew. Chem. Int. Ed. 2013, 52, 3259-3263.

9. SAINT Software User's Guide, Version 7.34a ed.; Bruker AXS Inc.: Madison, WI, 2005.

10. Sheldrick, G. M., SADABS, the Siemens Area Detector Absorption Correction; University of Göttingen: Göttingen, Germany, 2005.

11. Sheldrick, G. M., TWINABS, the Siemens Area Detector Absorption Correction; University of Göttingen: Göttingen, Germany, 2005.

12. Sarsfield, M. J.; Helliwell, M.; Collison, D., The first uranyl-methine carbon bond; a complex with out-of-plane uranyl equatorial coordination. Chem. Commun. 2002, 2264-2265.

13. Piarulli, U.; Solari, E.; Floriani, C.; Chiesi-Villa, A.; Rizzoli, C., Redox Chemistry Associated with the Complexation of Vanadium(V) and Tungsten(VI) by meso-Octaethylporphyrinogen: Formation and Cleavage of Cyclopropane Units Functioning as Shuttles of Two Electrons. J. Am. Chem. Soc. 1996, 118, 3634-3642.

14. Bachmann, J.; Nocera, D. G., Multielectron Chemistry of Zinc Porphyrinogen: A LigandBased Platform for Two-Electron Mixed Valency. J. Am. Chem. Soc. 2004, 126, 2829-2837. 